\title{
An a priori DNS study of the shadow-position mixing model
}

\author{
X. -Y. Zhao ${ }^{\text {a }}$ A. Bhagatwala ${ }^{\text {b }}$, J. H. Chen ${ }^{\text {b }}$, D. C. Haworth ${ }^{\text {c }}$, S.B. Pope ${ }^{\text {d }}$ \\ ${ }^{a}$ Department of Mechanical Engineering, \\ University of Connecticut, \\ Storrs, CT 06269 \\ ${ }^{b}$ Combustion Research Facility, \\ Sandia National Laboratories, \\ Livermore, CA 94550 \\ ${ }^{c}$ Department of Mechanical and Nuclear Engineering, \\ The Pennsylvania State University, \\ University Park, PA 16802 \\ ${ }^{d}$ Sibley School of Mechanical and Aerospace Engineering, \\ Cornell University, \\ Ithaca, NY 14853
}

\begin{abstract}
The modeling of mixing by molecular diffusion is a central aspect for transported probability density function (tPDF) methods. In this paper, the newly-proposed shadow position mixing model (SPMM) is examined, using a DNS database for a temporally evolving di-methyl ether slot jet flame. Two methods that invoke different levels of approximation are proposed to extract the shadow displacement (equivalent to shadow position) from the DNS database. An approach for a priori analysis of the mixing-model performance is developed. The shadow displacement is highly correlated with both mixture fraction and velocity, and the peak correlation coefficient of the shadow displacement and mixture fraction is higher than that of the shadow displacement and velocity. This suggests that the composition-space localness is reasonably well enforced by the model, with appropriate choices of model constants. The conditional diffusion of mixture fraction and major species from DNS and from SPMM are then compared, using mixing rates that are derived by matching the mixture fraction scalar dissipation rates. Good qualitative agreement is found, for the prediction of the locations of zero and maximum/minimum conditional diffusion locations for mixture fraction and individual species. Similar comparisons are performed for DNS and the IECM (interaction by exchange with the conditional mean) model. The agreement between SPMM and DNS is better than that between IECM and DNS, in terms of conditional diffusion iso-contour similarities and global normalized residual levels. It is found that a suitable value for the model constant $c$ that controls the mixing frequency can be derived using the local normalized scalar variance, and that

Preprint submitted to Combustion and Flame

December 10, 2015
\end{abstract}

(C) 2015. This manuscript version is made available under the Elsevier user license http://www.elsevier.com/open-access/userlicense/1.0/ 
the model constant $a$ controls the localness of the model. A higher-Reynolds-number test case is anticipated to be more appropriate to evaluate the mixing models, and stand-alone transported PDF simulations are required to more fully enforce localness and to assess model performance.

\section{Introduction}

The modeling of mixing by molecular diffusion of chemical species is a central aspect of turbulent combustion modeling. Molecular mixing models play an especially important role in the transported probability density function (tPDF) method, since the chemical reaction source terms are treated exactly, so that molecular transport is the dominant modeling burden. Enormous effort has been invested into the development of molecular mixing models over the past few decades [1]. Available mixing models include the coalescence and dispersion model (CD, or Curl's model) [2], a modified Curl's model [3] (MCD), the interaction by exchange with the mean (IEM) model [4] (or equivalently, the linear mean square estimation - LSME - model [5]), the interaction by exchange with the conditional mean (IECM) model [6, 7, 8], mapping closure (MC) models [9, 10], multiple mapping closure (MMC) models [11, 12], the Euclidean minimum spanning tree (EMST) model [13], FokkerPlanck (FP)-equation based models [14, 15], and the parameterization of one-dimensional scalar profiles (PSPs) mixing model $[16,17,18]$.

Criteria for assessing mixing models and desirable properties of mixing models have been established $[13,19]$, and these are summarized in Table 1 . The attributes of four of the more prominent mixing models, and the model that is the focus of the present study, with respect to these criteria are indicated in Table 1. None of the existing models possesses all of the desirable properties, although some models (e.g., EMST) satisfy the most important criteria, which include conservation of means, localness and boundedness. EMST has proven to be robust and accurate over a wide range of conditions [20]. Nevertheless, EMST has limitations. For example, it violates the linearity and independence principles, it does not yield Gaussian PDFs in appropriate circumstances, and it is prone to stranding of particles in composition space [13, 21]. As a particle-based method, there is no proof that EMST converges to a limit as the number of particles tends to infinity.

A new mixing model - the shadow-position mixing model (SPMM) - was proposed recently by Pope [21]. SPMM draws on knowledge from and experience with the existing mixing models as illustrated in Fig. 1. For example, in the IECM model it is argued that fluid particles with the same velocity have a higher probability of mixing with one another, and because of this the IECM 
model satisfies turbulent-dispersion theory. On the other hand, the localness in composition space enforced in the EMST model has led to great success in capturing the effects of turbulence-chemistry interactions. The central idea behind the creation of SPMM is that fluid particles that share similar history should have a higher probability of mixing with one another. Here, the word "history" implies not only velocity history as captured by the IECM model, but also the scalar mixing history as suggested by the EMST model. Thus, by construction, SPMM (to a good approximation) satisfies criteria I to VII in Table 1 [21], which makes it a potentially attractive mixing model. It has already been demonstrated that SPMM can preserve the localness in composition space (leading to strong burning) in a homogeneous isotropic reactive scalar mixing layer [21], while the IECM model leads to local extinction. A recent a posteriori study [22] of a temporally-evolving syngas flame also shows that SPMM can enforce localness in composition space to the same extent that the EMST model does.

In SPMM, the evolution of the composition of a notional Lagrangian particle (that is intended to model the behavior of a fluid particle) due to molecular mixing is described by the following equation:

$$
\frac{d \phi^{*}}{d t}=-\frac{c}{T_{L}}\left(\phi^{*}-\left\langle\phi^{*} \mid \mathbf{Z}^{*}, \mathbf{X}^{*}\right\rangle_{\rho}\right)
$$

where $c$ is a model constant, $\mathbf{X}^{*}$ and $\phi^{*}$ are the particle position and composition, respectively, and $\left\langle\phi^{*} \mid \mathbf{Z}^{*}, \mathbf{X}^{*}\right\rangle_{\rho}$ denotes the Favre average of $\phi^{*}$ conditioned on $\mathbf{Z}^{*}$ and $\mathbf{X}^{*}$. The particle position evolves by $d \mathbf{X}^{*} / d t=\mathbf{U}^{*}$, where $\mathbf{U}^{*}$ is the particle velocity. The quantity $\mathbf{Z}^{*}$ is the shadow position, which is a non-physical quantity associated with the particle, where $\mathbf{Z}^{*}$ evolves by the stochastic differential equation:

$$
d \mathbf{Z}^{*}=\left\langle\mathbf{U}^{*} \mid \mathbf{Z}^{*}\right\rangle_{\rho} d t-\frac{a}{T_{L}}\left(\mathbf{Z}^{*}-\mathbf{X}^{*}\right) d t+b\left(2 \sigma^{2} T_{L}\right)^{1 / 2} d \mathbf{W}^{\prime} .
$$

Here $a$ and $b$ are model constants, $T_{L}$ is a Lagrangian time scale, $\sigma$ is the r.m.s. velocity and $\mathbf{W}^{\prime}$ is an isotropic Wiener process: the prime is used to emphasize that the Wiener process in the shadow position equation is independent of the Wiener process $\mathbf{W}$ that is associated with particle motion.

The shadow displacement $\mathbf{R}^{*}$ is then introduced, which is defined as

$$
\mathbf{R}^{*} \equiv \mathbf{Z}^{*}-\mathbf{X}^{*}
$$

The evolution of $\mathbf{R}^{*}$ can be derived from Eqs. 2 and 3 as:

$$
d \mathbf{R}^{*}=-\left(\mathbf{U}^{*}-\left\langle\mathbf{U}^{*} \mid \mathbf{X}^{*}\right\rangle_{\rho}\right) d t-\frac{a}{T_{L}} \mathbf{R}^{*} d t+b\left(2 \sigma^{2} T_{L}\right)^{1 / 2} d \mathbf{W}^{\prime} .
$$


Recognizing that conditioning on $\left(\mathbf{Z}^{*}, \mathbf{X}^{*}\right)$ is equivalent to conditioning on $\left(\mathbf{R}^{*}, \mathbf{X}^{*}\right)$, one can reformulate SPMM as,

$$
\frac{d \phi^{*}}{d t}=-\frac{c}{T_{L}}\left(\phi^{*}-\left\langle\phi^{*} \mid \mathbf{R}^{*}, \mathbf{X}^{*}\right\rangle_{\rho}\right) \quad(\mathrm{SPMM})
$$

SPMM is similar in structure to the IEM and IECM mixing models, for which the particle composition evolution equations corresponding to Eq. 5 can be written as,

$$
\begin{aligned}
& \frac{d \phi^{*}}{d t}=-\frac{c_{\phi}}{T_{L}}\left(\phi^{*}-\left\langle\phi^{*} \mid \mathbf{X}^{*}\right\rangle_{\rho}\right) \quad(\mathrm{IEM}), \\
& \frac{d \phi^{*}}{d t}=-\frac{c_{U}}{T_{L}}\left(\phi^{*}-\left\langle\phi^{*} \mid \mathbf{U}^{*}, \mathbf{X}^{*}\right\rangle_{\rho}\right) \quad(\text { IECM })
\end{aligned}
$$

In all three models, the particle composition relaxes toward the local (conditional) mean composition on a time scale $T_{L}$. The choice of the conditioning variable $\left(\mathbf{R}^{*}\right.$ or $\left.\mathbf{U}^{*}\right)$ controls the location of zero conditional diffusion points/lines in composition space; this is discussed further in Sec. 4 below. The choice of conditioning variable has been guided by insight gained from direct numerical simulations (DNS) and experimental studies of canonical nonreacting and reacting turbulent flows. For example, the observation that the mean scalar diffusion conditioned on the velocity is linearly related to the velocity [23] motivates the choice of particle velocity as a conditioning variable in IECM. A physical interpretation is that molecular mixing can only take place between fluid particles that are sufficiently close (on the order of a Kolmogorov scale of separation) in physical space, and therefore that reside in the same turbulent eddy and have similar velocities [7]. The second key element in all three models is the mixing timescale, which controls the rate at which the composition relaxes to its local (conditional) mean value. Different expressions for the mixing rate have been used for different model variants. For example, the term $c_{\phi} / T_{L}$ in the IEM model (Eq. 6) is more commonly written as $c_{\phi} \omega / 2$, where $\omega$ is a turbulent frequency (e.g., $\omega=\varepsilon / k$ in a Reynolds-averaged formulation, where $k$ is the turbulence kinetic energy and $\varepsilon$ is the viscous dissipation rate of turbulence kinetic energy) and $c_{\phi}$ is a model constant that can be identified as the ratio of a turbulence timescale $\tau=1 / \omega$ to a turbulent scalar timescale $\tau_{\phi}$, with $c_{\phi}=2.0$ usually taken as the standard value.

In the case of SPMM, the physical interpretation of the conditioning variable $\mathbf{R}^{*}$ is less clear, although $\mathbf{R}^{*}$ is, by design, highly correlated with both velocity and scalars [21]. Moreover, model analysis/calibration to date has been limited to highly idealized configurations (homogeneous, isotropic, 
constant-property systems [21]), and the extent to which the conclusions that were drawn there regarding specifications of an appropriate mixing timescale and model constants can be generalized is not clear. It is desirable to develop a better physical understanding of the shadow displacement $\mathbf{R}^{*}$, to establish protocols for how to extract $\mathbf{R}^{*}$ from DNS or experimental data, and to test/extend the model for variable-property, inhomogeneous reacting systems with more realistic turbulence fields. All of these are prerequisites to applying the model effectively in Reynolds-averaged and/or largeeddy simulations of turbulent reacting flows. In addition, the potential computational difficulty of conditioning on a three-dimensional variable (i.e., $\mathbf{R}^{*}$ ) requires the identification of a preferred direction in the test flame, and conditioning only on the component of $\mathbf{R}^{*}$ in that direction. With these motivations and limitations in mind, an a priori study of SPMM using DNS is undertaken here. The configuration is a temporally evolving di-methyl ether (DME) slot jet flame [24]. This configuration features highly anisotropic and nonstationary turbulence, one direction of spatial inhomogeneity, and strong turbulence-chemistry interactions (strong local extinction and reignition), making it a challenging and appropriate target for testing mixing models.

The remainder of the paper is organized as follows. Details of the DNS configuration are provided in Sec. 2. There Favre-averaged velocity and scalar statistics are extracted as functions of time, and the Lagrangian time scale $T_{L}$ that is required in SPMM is discussed and quantified. In Sec. 3, two different methods for extracting $\mathbf{R}^{*}$ are proposed, and the evaluation of conditional diffusion is explained in detail. Results are then presented and discussed in Sec. 4. The physical meaning of shadow displacement is elucidated through its structure in physical space and its relationships with velocity and mixture fraction. Comparisons of conditional diffusion between DNS and SPMM and specification of model constants are presented and discussed. Finally, a summary and conclusions are provided in Sec. 5 .

\section{The test flame}

In this section, the DNS configuration is presented first. Velocity and scalar statistics including turbulent transport coefficients and Lagrangian time scales are then presented, and issues in extracting appropriately smooth profiles from noisy DNS data are addressed. In addition to providing input that is required for SPMM, the flow statistics provide information on the structure of the system that is needed for subsequent modeling studies. 


\subsection{DNS configuration}

A statistically one-dimensional, temporally evolving DME slot jet flame is considered [24] (Fig. 2). The computational domain extends $10 H$ in the stream-wise direction $(x), 16 H$ in the transverse direction $(y)$, and $8 H$ in the span-wise direction $(z)$, where $H$ is the width of the central fuel jet $(H=2.54 \mathrm{~mm})$. Periodic boundary conditions are imposed in the $x$ and $z$ directions, and outflow boundary conditions are specified in $y$. The system is statistically homogeneous in the $x$ and $z$ directions. A central fuel jet ( $12 \% \mathrm{DME}, 18 \% \mathrm{H}_{2}$ and $70 \% \mathrm{~N}_{2}$ by volume) is injected in the $x$ direction. The fuel jet is sandwiched between two streams of oxidizer $\left(31 \% \mathrm{O}_{2}\right.$ and $69 \% \mathrm{~N}_{2}$ by volume) injected in the opposite direction. To initialize the flow field, small isotropic broadband turbulent velocity fluctuations are superimposed on the mean velocity field, with the fluctuations filtered outside the central jet. Thus, the computational domain can be divided into two regions: the inner reactive turbulent flame core, which is located at the center of the computational domain in $y$ and grows with time; and the outer inert coflow regions with near-zero turbulent fluctuations.

The Reynolds number based on the fuel-jet properties is $R e \equiv U_{j} D_{H} / \nu=13,050$, where $D_{H}$ is the hydraulic diameter defined as $D_{H}=2 L_{z} H /\left(L_{z}+H\right)$ and $L_{z}$ is the spanwise extent of the domain, $\nu$ is the kinematic viscosity of the unburnt fuel mixture at inlet temperature $(450 \mathrm{~K})$, and $U_{j}$ is given by $U_{\text {fuel }}-U_{\text {oxidizer }}\left(\left|U_{\text {fuel }}\right|=\left|U_{\text {oxidizer }}\right|\right)$ based on initial conditions. The Damköhler number is defined to be $D a=\chi_{q} t_{j}$, where $t_{j}$ is the mean jet convective time $\left(t_{j}=D_{H} / U_{j}\right)$ estimated to be $41 \mu \mathrm{s}$ based on the initial conditions, and $\chi_{q}$ is the extinction scalar dissipation rate that

is $1950 \mathrm{~s}^{-1}$. This combination of $\chi_{q}$ and $t_{j}$ results in a Damköhler number of 0.08. This lowDamköhler-number flame exhibits a significant degree of local extinction and reignition. Similar configurations have been studied using DNS with syngas and ethylene fuels [25, 26], at different Reynolds and Damköhler numbers. The Reynolds number of this flame is slightly higher than those in previous studies, and the Damköhler number is chosen to ensure significant occurrence of local extinction.

A 30-species reduced DME mechanism [24] is used. The grid size is uniform in all three directions, and it is chosen to be of the same order as the initial Kolmogorov length scale $\eta$. With the decay of turbulence, the grid size is expected to correspond to even a smaller fraction of the local Kolmogorov length scale. The time interval between adjacent DNS solution snapshots that are available for analysis is approximately three times the local Kolmogorov time scale $t_{\eta}$ during the initial period of the flame, and is reduced to $0.25 t_{\eta}$ at later times. Therefore, both the spatial 
and temporal resolutions resolve the Kolmogorov scales, which are sufficient for the purposes of this a priori study. Reynolds-averaged (or Favre-averaged) mean quantities are estimated as averages over grid points in $x-z$ planes, and are functions of $y$ and $t$ only. However, because of the finite sample size, some care needs to be taken in extracting mean quantities, and this is discussed further below.

A global indication of the robustness of combustion is the burning fraction, which is defined as the fraction of points on the instantaneous three-dimensional isosurface of stoichiometric mixture fraction (here $\xi_{s t}=0.375$ ) where the local $\mathrm{OH}$ mass fraction is above $75 \%$ of the peak $\mathrm{OH}$ mass fraction (i.e., $9.77 \times 10^{-4}$ ).

The evolution of the burning fraction with time is shown in Fig. 3. There unity denotes robust burning, and a value of zero corresponds to total extinction. It can be seen that the initially robust DME flame undergoes strong local extinction, followed by reignition. The three time instants labeled as $t_{B M E}, t_{M E}$, and $t_{R I}$ in Fig. 3 represent times before, at, and after maximum local extinction; most of the subsequent analysis is performed at these three instants.

\subsection{Velocity and mixture-fraction statistics}

By construction, the conditioning variable $\mathbf{R}^{*}$ is correlated with both the velocity and scalar fields; this becomes more evident in the subsequent analysis (Secs. 3 and 4). Here key velocity- and scalar-field statistics that are needed for model formulation and validation are presented. These also provide useful insight into the structure of this statistically one-dimensional, time-dependent flame, which is useful for subsequent modeling studies.

Figure 4 shows the Favre-averaged mean and r.m.s. velocity components and mixture fraction profiles at the three time instants indicated in Fig. 3. Here $u, v$ and $w$ denote the $x, y$ and $z$ components of velocity, respectively, and the mixture fraction $\xi$ is defined using Bilger's formula [27]. Favre-averaged quantities are denoted using a tilde, and fluctuations with respect to Favre-averaged mean values are denoted using a double prime. For these mean profiles, statistical symmetry (or anti-symmetry, as appropriate) with respect to the center plane in $y$ has been assumed to double the sample size and reduce noise. Even with that, some statistical error can still be observed (e.g., non-zero values of $\widetilde{w})$.

By design, the stoichiometric surface for the flame lies in the high-shear/high-turbulence region,

so that the peak of $\xi_{r m s} \equiv \sqrt{\widetilde{\xi^{\prime \prime 2}}}$ approximately aligns with the peaks of $u_{r m s} \equiv \sqrt{\widetilde{u^{\prime \prime 2}}}, v_{r m s} \equiv \sqrt{\widetilde{v^{\prime \prime 2}}}$, 
and $w_{r m s} \equiv \sqrt{\overline{w^{\prime \prime 2}}}$ in Fig. 4. The r.m.s. of both velocity and mixture fraction go to zero in the quiescent coflow regions. Here a cutoff at $10 \%$ of $\xi_{r m s, \max }[25]$ is used to distinguish between the active combustion region and the nonreactive coflow. That is, at locations where $\xi_{r m s}<0.1 \xi_{r m s, \max }$ (hereafter referred to as the coflow), the turbulent fluctuations are small and the composition is essentially that of the oxidizer stream (verified by histograms of mixture fraction at those locations). In regions where $\xi_{r m s} \geq 0.1 \xi_{r m s, \max }$ (hereafter referred to as the flame core), turbulence is intense, and the mixture is reactive.

Profiles of the components of the normalized anisotropy tensor $\left(b_{i j}=\overline{u_{i}^{\prime \prime} u_{j}^{\prime \prime}} \overline{u_{k}^{\prime \prime} u_{k}^{\prime \prime}}-\frac{1}{3} \delta_{i j}\right)$ at the same three time instants are shown in Fig. 5. It can be seen that the flow is highly anisotropic inside the flame core. Also shown are the profiles corresponding to a standard $k-\varepsilon$ turbulence model, where

$$
\left.\widetilde{u_{i}^{\prime \prime} u_{j}^{\prime \prime}}\right|_{k-\varepsilon}=-\nu_{T}\left(\frac{\partial \widetilde{u}_{i}}{\partial x_{j}}+\frac{\partial \widetilde{u}_{j}}{\partial x_{i}}\right)+\frac{2}{3} \nu_{T} \frac{\partial \widetilde{u}_{k}}{\partial x_{k}} \delta_{i j}+\frac{2}{3} k \delta_{i j} .
$$

Here the compact notation $u_{i}$ is used to represent the three components of velocity, i.e., $u_{1}=u$, $u_{2}=v, u_{3}=w$. The turbulent viscosity is modeled by $\nu_{T}=C_{\mu} k^{2} / \varepsilon$, with $C_{\mu}=0.09$. $\varepsilon$ is defined as $\left\langle 2 \nu s_{i j} s_{i j}\right\rangle_{\rho}$, where $s_{i j}$ is the turbulent strain rate tensor $\left(s_{i j}=\frac{1}{2}\left(\frac{\partial u_{i}{ }^{\prime \prime}}{\partial x_{j}}+\frac{\partial u_{j}{ }^{\prime \prime}}{\partial x_{i}}\right)\right)$. For the reactive mixture, this model still performs reasonably well for the shear stress (represented by $b_{12}$ ). The highly anisotropic and nonstationary nature of this configuration is very different from the idealized configurations considered in [21], and presents a significant modeling challenge for SPMM or any other mixing model.

Finally, the turbulent mixture-fraction fluxes $\left(\widetilde{u^{\prime \prime} \xi^{\prime \prime}}, \widetilde{v^{\prime \prime} \xi^{\prime \prime}}, \widetilde{w^{\prime \prime} \xi^{\prime \prime}}\right)$ extracted from the DNS are shown in Fig. 6. Both $\overline{u^{\prime \prime} \xi^{\prime \prime}}$ and $\overline{v^{\prime \prime} \xi^{\prime \prime}}$ are non-zero at all time instants, and the magnitude of $\overline{u^{\prime \prime} \xi^{\prime \prime}}$ is larger than that of $\widetilde{v^{\prime \prime} \xi^{\prime \prime}}$. The $\overline{w^{\prime \prime} \xi^{\prime \prime}}$ profiles fluctuate around zero. A similar relationship between $\widetilde{u^{\prime \prime} \xi^{\prime \prime}}$ and $\widetilde{v^{\prime \prime} \xi^{\prime \prime}}$ was observed experimentally in a constant-gradient homogeneous shear flow with temperature as a passive scalar $[28,29]$. For the temporally evolving slot-jet flame, the direction of the scalar flux is significantly different than that of the mean scalar gradient $(\partial \widetilde{\xi} / \partial x=0$ here).

\subsection{Evaluation of the Lagrangian time scale $T_{L}$}

Specification of an appropriate time scale is essential for any mixing model (e.g., Eqs. 5, 6, and 7), and is particularly important in the context of SPMM for the shadow displacement equation (Eq. 4). A Lagrangian time scale $T_{L}$ can be defined unambiguously in terms of the two-time Lagrangian velocity autocorrelation function in statistically stationary, homogeneous, isotropic turbulence [21, 30]. 
However, the definition for Lagrangian time scales is not obvious in a temporally-evolving, inhomogeneous, and anisotropic reacting flow. To be used in a modeling study, the definition must be in terms of quantities that are included in standard models. Borrowing from the concepts developed in statistically stationary, homogeneous, isotropic turbulence [21], for this case, we propose three approximations for the Lagrangian time scales, as listed in Eqs. 9, 10 and 11:

$$
\begin{array}{rr}
T_{L, 1}=-\widetilde{v^{\prime \prime} \xi^{\prime \prime}} /\left(\sigma^{2} \frac{\partial \widetilde{\xi}}{\partial y}\right) & (\text { Approximation } 1), \\
T_{L, 2}=C_{1}(t) L(y, t) / \sigma=C_{1}(t) k^{2 / 3} /(\sigma \varepsilon) & (\text { Approximation } 2), \\
T_{L, 3}=C_{2} k / \varepsilon & (\text { Approximation } 3) .
\end{array}
$$

Here, $\sigma^{2}$ is the $y$-direction velocity variance $\left(\sigma^{2}=\widetilde{v^{\prime \prime 2}}\right), C_{1}(t)$ is a time-dependent coefficient, $L(y, t)$ is a length scale, and $C_{2}$ is a time-independent constant. The turbulent scalar flux $\left(\overline{v^{\prime \prime} \xi^{\prime \prime}}\right)$ can be expressed using $T_{L}$ by rearranging Eq. 9:

$$
\widetilde{v^{\prime \prime} \xi^{\prime \prime}}=-T_{L} \sigma^{2} \frac{\partial \widetilde{\xi}}{\partial y}
$$

Both Approximation 1 and Approximation 2 attempt to match the turbulent scalar flux calculated using $T_{L}$ to that obtained directly from DNS. All of the quantities required in Approximation 1 (Eq. 9) are available from the DNS (Sec. 2.2). For Approximation 2, $C_{1}(t)$ is a time-varying constant that is obtained by minimizing (in a least-squares sense over $y$ ) the difference between the scalar flux obtained from DNS and that obtained from Eq. 12 using $T_{L, 2}$. The turbulent length scale $L(y, t)$ is specified to be $L(y, t)=C_{\mu}^{3 / 4} k(y, t)^{3 / 2} / \varepsilon(y, t)$, using definitions borrowed from the $k$ - $\varepsilon$ model. For $6 t_{j}, 8 t_{j}$ and $14 t_{j}, C_{1}$ is found to be $0.58,0.72$, and 0.99 , respectively. For Approx $3, C_{2}$ is derived to be $C_{2}=\left(\frac{1}{2}+\frac{3}{4} C_{0}\right)^{-1}$ according to the Langevin model of [21]. Here $C_{0}$ can be taken as a constant value of 2.1, or it can be Reynolds-number dependent [31]. Approximation 3 is included here because it is much easier to obtain $T_{L, 3}$ in a modeling study than to obtain $T_{L, 1}$ and $T_{L, 2}$ directly. Thus, it is of interest to compare the magnitude of $T_{L, 3}$ with $T_{L, 1}$ and $T_{L, 2}$. Since $\sigma^{2} T_{L}$ frequently appears as one term in the governing equations for SPMM (e.g., in Eq. 5), an apparent turbulent diffusivity $\Gamma_{T}$ is defined,

$$
\Gamma_{T}(y, t)=T_{L}(y, t) \sigma^{2}(y, t) .
$$

When obtained using different approximations of $T_{L}$, different apparent turbulent diffusivities are obtained (i.e., $\Gamma_{T, 1}, \Gamma_{T, 2}, \Gamma_{T, 3}$ ). 
It is important to provide smooth profiles of $T_{L}$ and $\Gamma_{T}$ to obtain reasonable distributions of the shadow displacement $\mathbf{R}^{*}$. A direct calculation using the profiles of $\widetilde{v^{\prime \prime} \xi^{\prime \prime}}, \partial \widetilde{\xi} / \partial y$ and $\sigma^{2}$ is poorly conditioned because of the division of two noisy profiles, both of which pass through zero, required in Approximation 1 (Eq. 9). To mitigate this, $\Gamma_{T, 1}$ is set to the mixture-averaged $\mathrm{N}_{2}$ molecular diffusivity and $T_{L, 1}$ is set to the jet time $t_{j}$ in regions where $\xi_{r m s} \leq 0.1 \xi_{r m s, \max }$ (outside of the flame core). Cross-validated cubic b-splines [32] are then used to smooth the profiles of $\Gamma_{T, 1}(y)$ at each time instant. The profiles of $T_{L, 2}$ and $T_{L, 3}$ are by construction adequately smooth, so no additional smoothing procedure is applied to $T_{L, 2}$ and $T_{L, 3}$ (hence $\Gamma_{T, 2}$ and $\Gamma_{T, 3}$ ).

Profiles of $T_{L, 1}$ and $T_{L, 2}$ and $T_{L, 3}$ are shown in Fig. 7. The profiles of $T_{L, 2}$ are smoother than those of $T_{L, 1}$, and they are also more uniform across the transverse direction. The profile of $T_{L, 3}$ is also quite smooth and uniform across the transverse direction, and the profiles of $T_{L, 3}$ and $T_{L, 2}$ generally enclose the profile of $T_{L, 1}$.

While the values of $T_{L, 1}$ and $T_{L, 2}$ are similar inside the reactive shear layers, they can be quite different at other locations. To further validate the approach, the turbulent scalar (mixture-fraction) flux $\left(\widetilde{v^{\prime \prime} \xi^{\prime \prime}}\right)$ extracted from DNS is compared with that obtained using Eq. 12 with either $T_{L, 1}$ or $T_{L, 2}$ in Fig. 8. The turbulent flux computed using $T_{L, 1}$ is closer to the DNS profiles, because it is calculated using the scalar flux extracted from DNS. Meanwhile, the scalar flux is recovered with acceptable accuracy using $T_{L, 2}$, in spite of the large differences between $T_{L, 1}$ and $T_{L, 2}$ shown in Fig. 7 near the centerline. This is because $\overline{v^{\prime \prime} \xi^{\prime \prime}}$ is essentially zero near the centerline, and the differences between $T_{L, 1}$ and $T_{L, 2}$ in that region make insignificant differences in the scalar flux. Inside the shear layer where the scalar gradient is significant, $T_{L, 1}$ and $T_{L, 2}$ are quite close. The milder variation in the $y$-direction of $T_{L, 2}$ favors the use of $T_{L, 2}$ in the integration of the ODEs required later in calculating $R^{*}$.

It should be pointed out here that the Lagrangian time scale extracted in this section is not the same as the turbulence integral time scale. For example, by launching tracer particles at locations where the transverse r.m.s. velocity peaks, the auto-correlation function of velocity has been calculated and fitted to the Sawford model [33]. The inferred integral time scale for $t=8 t_{j}$ is approximately $3.9 \times 10^{-5} \mathrm{~s}$, which is approximately 1.5 times $T_{L, 2}$, and $T_{L, 2}$ is closer to the value calculated from the Lagrangian tracer particles than $T_{L, 3}$. To make $T_{L, 3}$ approximately equal to $T_{L, 2}$, the implied $C_{0}$ values are calculated to be $10.5,8.2$, and 5.7 at $6 t_{j}, 8 t_{j}$ and $14 t_{j}$, respectively. Given the similarity between $T_{L, 3}$ and $T_{L, 2}$, it is expected that it is possible to use $T_{L, 3}$ in a modeling 
study with appropriate scaling factor or with a proper model for a Reynolds-number-dependent $C_{0}$.

Based on the analysis above, Approximation 2 is adopted to extract $\Gamma_{T}$ and $T_{L}$ in the remainder of this paper.

\section{Shadow displacement and conditional diffusion}

In this section, the challenge of evaluating the shadow displacement in a priori tests is discussed first, followed by the introduction of two different methods (Method 1 and Method 2) for extracting the shadow displacement from DNS. The specification of the three model constants $a, b$ and $c$ is then described. In Sec. 4, conditional scalar diffusion of mixture fraction and species mass fractions are examined to explain model behavior and to compare the model with DNS. Here the definition of the conditional diffusion is clarified, and the methods used to evaluate conditional diffusion from DNS and from SPMM (and other mixing models) are discussed.

\subsection{The challenge of the quantification of the shadow displacement $\mathbf{R}^{*}$ in a priori tests}

In an a posteriori simulation, the evolution of $\mathbf{R}^{*}$ is governed not only by Eq. 4 , but also by Eq. 5. The subtlety comes from the involvement of $\Gamma_{T}$ and $T_{L}$ in Eq. 4. Both quantities are defined using the scalar flux, the statistics of which can be altered through the evolution of scalar $\phi$ using Eq. 5. In other words, conditioning on $\mathbf{R}^{*}$ in Eq. 5 impacts the evolution of $\phi$, and the improved prediction of $\phi$ in turns changes the evolution of $\mathbf{R}^{*}$ through $\Gamma_{T}$ and $T_{L}$ in Eq. 4. The coupling between Eq. 4 and Eq. 5 is the driving mechanism that ensures localness in SPMM. It is recognized that the influence of Eq. 5 on the evolution of $\mathbf{R}^{*}$ cannot be directly represented in an a priori DNS test, because scalars are deterministic from the DNS solution and cannot be influenced by the introduction of $\mathbf{R}^{*}$.

To test the performance of SPMM, it is essential that $\mathbf{R}^{*}$ is properly correlated with the scalars to ensure localness. It is shown in [21] that the correlation coefficient of $\mathbf{R}^{*}$ and $\xi$ can be as high as unity, depending on the choice of model constants and the specific test case. Since the main objective of this study is to evaluate the performance of SPMM in terms of the prediction of conditional diffusion, indirect approaches (i.e., Method 1 and Method 2) that can best represent the tight coupling between $\mathbf{R}^{*}$ and $\xi$ for the current test flame are introduced. In Method $1, \mathbf{R}^{*}$ is obtained through solving Eq. 4, and the impact of Eq. 5 is introduced through a constructed quantity $X$. In Method 2, neither Eq. 4 nor Eq. 5 are used, and a surrogate of $\mathbf{R}^{*}$ is introduced, 
which is constructed based on correlation coefficients of velocity and mixture fraction. Methods 1 and 2 are discussed in detail in Secs. 3.2 and 3.3, respectively.

The flame considered here is statistically one dimensional, so that only the $y$ component of the shadow displacement vector $\mathbf{R}^{*}$ (denoted as $R^{*}$ ) is relevant. All the subsequent discussions on the shadow displacement are in the context of statistically one-dimensional flames.

\subsection{Quantification of the shadow displacement $\mathbf{R}^{*}$ : Method 1 (ODE integration)}

In Method 1, the shadow displacement $R^{*}$ is obtained through the following stochastic differential equation (i.e., the one dimensional form of Eq. 4),

$$
d R^{*}=-a R^{*} \frac{d t}{T_{L}}-v^{\prime \prime} d t+b\left(2 \sigma^{2} T_{L}\right)^{1 / 2} d W^{\prime} .
$$

The DNS velocity field is deterministic, and hence the only randomness in Eq. 14 is introduced by the Wiener process, $W^{\prime}$. Therefore, the PDF of $R^{*}$ obtained through Eq. 14 is Gaussian, and can be expressed in terms of its mean (denoted by $R$ ) and variance (denoted by $S$ ) [21]. Specifically, $R$ is defined to be $R=\left\langle R^{*}\right\rangle_{W}$, where \langle\rangle$_{W}$ denotes an average over the Wiener process. In this statistically one-dimensional, temporally evolving flame, the governing equations for $R$ and $S$ are [21]:

$$
\begin{gathered}
\frac{D R}{D t}=-\frac{a}{T_{L}} R-v^{\prime \prime}, \\
\frac{D S}{D t}=-\frac{2 a}{T_{L}} S+2 b^{2} \sigma^{2} T_{L},
\end{gathered}
$$

where the left-hand side is the usual material derivative (time-rate-of-change following a fluid particle).

From specified initial conditions, Eqs. 15 and 16 are integrated backward in time (first order) along fluid particle pathlines for an ensemble of imaginary particles located at each grid point at time $t$. A second-order backward integration has also been tested, and no discernable differences are observed. A trilinear interpolation scheme is used to calculate intermediate particle locations during the integration process. It has been reported that higher-order interpolation schemes are usually required to preserve the trajectory of the Lagrangian particles in turbulent flows [34]. However, since both the temporal and spatial resolutions are smaller than the Kolmogorov scales here, the second-order trilinear scheme should suffice for the current test flame. 
For any grid point $n$, with specified $R^{(n)}$ and $S^{(n)}$ values, $R^{*(n)}$ can be expressed as,

$$
R^{\star(n)}=R^{(n)}+\sqrt{S^{(n)}} g^{(n)} .
$$

The statistics of $R^{*}$ evolve the same way as Eq. 14, if $g$ is taken to be a standard Gaussian random variable. The correlation between $R^{\star}$ and $v$ is captured correctly. To represent the influence of Eq. 5 on the evolution of $R^{*}, g$ is expressed as a function of a new variable $X$, where $X$ is not correlated with $v$ and $R$, but is correlated with $\xi$. It should be noted that $\xi$ is not a unique choice to represent the scalars. However, it is a representative quantity, especially in non premixed flames where turbulent fluctuations of reacting quantities are strongly correlated with fluctuations of the mixture fraction [35]. Here a limiting case is considered, enforcing the highest possible correlation between $R^{*}$ and $\xi$ for a given model constant $a$ by constructing $X$ as:

$$
X \equiv[\xi]+\alpha[R]+\beta[v],
$$

where square brackets denote a standardized variable, e.g.,

$$
[\xi] \equiv(\xi-\tilde{\xi}) / \xi_{r m s} .
$$

Coefficients $\alpha$ and $\beta$ are determined by imposing the conditions that $X$ is uncorrelated with both $R$ and $v . g$ is then defined as

$$
g=\operatorname{sign}\left(-\rho_{v \xi}\right)[X]
$$

where $\rho_{v \xi}$ is the correlation coefficient between $v$ and $\xi$. By introducing the variable $X$, the correlation between $R^{*}$ and $\xi$ is maximized to represent the level of localness that SPMM can enforce in a corresponding a posteriori case, without changing the correlation between $R^{*}$ and $v$. The initial conditions for $R$ and $S$ are taken to be the corresponding statistically stationary solutions, described as:

$$
\begin{gathered}
R\left(x, y, z, t_{0}\right)=-\frac{v^{\prime \prime}\left(x, y, z, t_{0}\right) T_{L}\left(y, t_{0}\right)}{a+1}, \\
S\left(x, y, z, t_{0}\right)=\frac{\sigma^{2}\left(y, t_{0}\right) T_{L}^{2}\left(y, t_{0}\right) b^{2}}{a},
\end{gathered}
$$

The derivation of the statistically stationary solutions can be found in Appendix A. 


\subsection{Quantification of the shadow displacement $\mathbf{R}^{*}$ : Method 2 (Surrogate model)}

An alternative (potentially simpler) method to extract the shadow displacement $R^{*}$ from DNS or experiments was proposed recently in [36]. There, a surrogate for $R^{*}$ (denoted as $R_{s g}^{*}$ ) was constructed, which is a function of the correlation coefficients between $\xi, v$ and $R^{*}$. The correlation coefficients involving $R^{*}$ are specified to be functions of the correlation coefficient between $\xi$ and $v$ $\left(\rho_{v \xi}\right)$, as follows:

$$
\begin{array}{r}
\rho_{v R^{*}}=\rho_{v R^{*}}^{o}, \\
\rho_{R^{*} \xi}=\rho_{R^{*} \xi}^{o} \frac{\rho_{v \xi}}{\rho_{v \xi}^{o}} \text {, for }\left|\rho_{v \xi}\right| \leq \rho_{c r i t}, \\
\rho_{R^{*} \xi}=\rho_{v \xi} \rho_{v R^{*}}^{o}-\operatorname{sign}\left(\rho_{v \xi}\right)\left[\left(1-\rho_{v \xi}^{2}\right)\left(1-\rho_{v R^{*}}^{o 2}\right)\right]^{1 / 2}, \text { for }\left|\rho_{v \xi}\right|>\rho_{c r i t},
\end{array}
$$

where $\rho_{v R^{\star}}^{o}$ and $\rho_{R^{*} \xi}^{o}$ denote the values that were calculated for a statistically stationary, homoge-

neous, isotropic, uniform-mean-scalar-gradient example in [21]. Here $\rho_{c r i t} \equiv \frac{\rho_{v \xi}^{o}}{\rho_{R^{*} \xi}^{o}}$ is used to prevent non-realizable values of $\rho_{R^{*} \xi}$. The quantity $R_{s g}^{*}$ also depends on the standard deviation of velocity $\left(\sigma_{v}\right)$, the standard deviation of mixture fraction $\left(\sigma_{\xi}\right), v^{\prime \prime}$, and $\xi^{\prime \prime}$ at each grid point. The final form of $R_{s g}^{*}$ is:

$$
\frac{R_{s g}^{\star}}{\sigma_{R^{*}}}=\lambda \frac{v^{\prime \prime}}{\sigma_{v}}+\kappa \frac{\xi^{\prime \prime}}{\sigma_{\xi}}+\gamma \eta
$$

where $\lambda, \kappa$ and $\gamma$ are coefficients (which can be obtained in terms of $\rho_{R^{*} \xi}, \rho_{v R^{*}}$, with the condition $\left.\left\langle\left(\frac{R_{s g}^{*}}{\sigma_{R}^{*}}\right)^{2}\right\rangle=1\right)$, and $\eta$ is a standardized Gaussian random variable. Here $\sigma_{R^{*}}$ is the standard deviation of $R^{*}$, but the value of $\sigma_{R}^{*}$ is immaterial since $\frac{R_{s g}^{*}}{\sigma_{R}^{*}}$ is only used for conditioning.

To summarize, two different methods are introduced in this study to obtain the one-dimensional shadow displacement $\left(R^{*}\right)$ for the test flame. In Method 1, one solves time-evolving ODEs for the local mean $(R)$ and variance $(S)$ of shadow displacement $\left(R^{*}\right)$ with prescribed initial conditions. The correlation between $R^{*}$ and $\xi$ is ensured by introducing a variable $X$ that is maximally correlated with mixture fraction but uncorrelated with velocity. From here on, $R_{O D E}^{*}$ is denoted to represent $R^{*}$ obtained from Method 1. In Method 2, one replaces $R^{*}$ using the surrogate $R_{s g}^{*}$. Method 1 is more computationally intensive, but it also invokes fewer assumptions. Method 2 avoids integrating ODEs in a priori studies, which provides a simpler way to evaluate SPMM in DNS; this is also expedient in SPMM-based modeling studies, as one does not need to transport the shadow displacement as an additional variable. However, Method 2 invokes more assumptions, 
and the validity of those assumptions needs to be tested. In the present study, Method 1 is taken as the baseline, and comparisons with results from Method 2 are presented to assess the potential of Method 2.

Next, some additional discussion of the specification of model constants and the evaluation of conditional diffusion is provided.

\subsection{Definition of the conditional diffusion}

The performance of SPMM is evaluated by comparing the conditional diffusion extracted directly from DNS with that extracted from DNS according to the SPMM formulation. In this section, the conditional diffusion is defined and discussed.

For a composition variable $\phi$ (e.g., a mixture fraction or a species mass fraction), the molecular diffusive flux $\mathbf{J}^{\phi}$ is defined as:

$$
\begin{gathered}
\mathbf{J}^{\phi} \equiv-\rho \mathcal{D}_{\phi} \nabla \phi(\text { for mixture fraction) }, \\
\mathbf{J}^{\phi} \equiv-\rho \mathcal{D}_{\phi}\left(\nabla Y_{i}+\frac{Y_{i}}{M} \nabla M\right) \text { (for species) },
\end{gathered}
$$

where $\mathcal{D}_{\phi}$ is either the thermal diffusivity (for mixture fraction) or the mixture-averaged molecular diffusivity of composition variable $\phi$, and $M$ is the mixture-averaged molecular weight. In modeling studies, the mean diffusive flux $\mathcal{J}^{\phi}$ is usually defined by replacing the values of $\rho, \mathcal{D}_{\phi}$ and $\phi$ in Eqs. 27 and 28 by their corresponding mean values:

$$
\begin{array}{r}
\mathcal{J}^{\phi} \equiv-\langle\rho\rangle \widetilde{\mathcal{D}}_{\phi} \nabla \widetilde{\phi} \text { (for mixture fraction) }, \\
\mathcal{J}^{\phi} \equiv-\langle\rho\rangle \widetilde{\mathcal{D}}_{\phi}\left(\nabla \tilde{Y}_{i}+\frac{\tilde{Y}_{i}}{\langle M\rangle} \nabla\langle M\rangle\right) \text { (for species) } .
\end{array}
$$

With these definitions, $\left\langle\mathbf{J}^{\phi}\right\rangle \neq \mathcal{J}^{\phi}$ for the variable-property case, although in high-Reynolds-number turbulent systems, $\left\langle\mathbf{J}^{\phi}\right\rangle, \mathcal{J}^{\phi}$, and their difference should all be small compared to the r.m.s. scalar flux. The part of the diffusive flux represented by mixing models is

$$
\mathbf{J}^{\phi} \equiv \mathbf{J}^{\phi}-\mathcal{J}^{\phi}
$$

Note that the mean $-\left\langle\mathbf{J}^{\prime}\right\rangle$ is, in general, non-zero, although it is zero in models.

In the DNS, the rate of change of $\phi$ due to $\mathbf{J}^{\prime \phi}$ is

$$
\left(\frac{\partial \phi}{\partial t}\right)_{D N S}=-\frac{1}{\rho} \nabla \cdot \mathbf{J}^{\prime \phi}
$$


whereas the rate of change of the particle composition due to the SPMM model is

$$
\left(\frac{d \phi}{d t}\right)_{S P M M}=-\frac{c}{T_{L}}\left(\psi-\left\langle\phi(\mathbf{x}, t) \mid \mathbf{X}^{*}(t)=\mathbf{x}, \mathbf{R}^{*}(t)=\mathbf{R}\right\rangle_{\rho}\right) .
$$

We define the conditional diffusions to be the conditioned means of these quantities $\left(\left(\frac{\partial \phi}{\partial t}\right)_{D N S}\right.$ and $\left.\left(\frac{\partial \phi}{\partial t}\right)_{S P M M}\right)$, i.e.,

$$
\begin{gathered}
D^{S P M M}(\psi, \mathbf{x}, \mathbf{R}, t) \equiv\left\langle\left(\frac{\partial \phi}{\partial t}\right)_{D N S} \mid \phi=\psi, \mathbf{X}^{*}(t)=\mathbf{x}, \mathbf{R}^{*}(t)=\mathbf{R}\right\rangle, \\
D_{M}^{S P M M}(\psi, \mathbf{x}, \mathbf{R}, t) \equiv\left\langle\left(\frac{d \phi}{d t}\right)_{S P M M} \mid \phi=\psi, \mathbf{X}^{*}(t)=\mathbf{x}, \mathbf{R}^{*}(t)=\mathbf{R}\right\rangle .
\end{gathered}
$$

Here the subscript $M$ is used to distinguish the conditional diffusion corresponding to the model from that corresponding directly to the DNS conditional diffusion. If $D_{M}^{S P M M}=D^{S P M M}$, then the SPMM is perfect in the sense that it causes the joint PDF of $\phi$ to evolve correctly. Hence we appraise the model by comparing these two quantities. (Note that $D_{M}^{S P M M}=D^{S P M M}$ is a necessary and sufficient condition, and a weaker condition than $\left(\frac{\partial \phi}{\partial t}\right)_{D N S}=\left(\frac{\partial \phi}{\partial t}\right)_{S P M M}$, which is impossible for a model to satisfy.)

For the IECM and IEM models, similar definitions are given by,

$$
\begin{array}{r}
\left(\frac{d \phi}{d t}\right)_{I E C M}=-\frac{c_{U}}{T_{L}}\left(\psi-\left\langle\phi(\mathbf{x}, t) \mid \mathbf{X}^{*}(t)=\mathbf{x}, \mathbf{U}^{*}(t)=\mathbf{u}\right\rangle_{\rho}\right), \\
\left(\frac{d \phi}{d t}\right)_{I E M}=-\frac{c_{\phi}}{T_{L}}\left(\psi-\left\langle\phi(\mathbf{x}, t) \mid \mathbf{X}^{*}(t)=\mathbf{x}\right\rangle_{\rho}\right) .
\end{array}
$$

For subsequent analysis and comparisons, the corresponding definitions for the IECM and IEM mixing models are:

$$
\begin{gathered}
D^{I E C M}(\psi, \mathbf{x}, \mathbf{V}, t) \equiv\left\langle\left(\frac{\partial \phi}{\partial t}\right)_{D N S} \mid \phi=\psi, \mathbf{X}^{*}(t)=\mathbf{x}, \mathbf{U}^{*}(t)=\mathbf{u}\right\rangle \\
D_{M}^{I E C M}(\psi, \mathbf{x}, \mathbf{V}, t) \equiv\left\langle\left(\frac{d \phi}{d t}\right)_{I E C M} \mid \phi=\psi, \mathbf{X}^{*}(t)=\mathbf{x}, \mathbf{U}^{*}(t)=\mathbf{u}\right\rangle \\
D^{I E M}(\psi, \mathbf{x}, t) \equiv\left\langle\left(\frac{\partial \phi}{\partial t}\right)_{D N S} \mid \phi=\psi, \mathbf{X}^{*}(t)=\mathbf{x}\right\rangle \\
D_{M}^{I E M}(\psi, \mathbf{x}, t) \equiv\left\langle\left(\frac{d \phi}{d t}\right)_{I E M} \mid \phi=\psi, \mathbf{X}^{*}(t)=\mathbf{x}\right\rangle
\end{gathered}
$$

It is noted that Eqs. 34, 38, 40 take the same form, except that the conditioning variables are different.

In the current configuration, the Reynolds number is moderate, and it is of interest to check the magnitudes of the mean and standard derivations of $\left(\frac{\partial \phi}{\partial t}\right)_{D}=-\frac{1}{\rho} \nabla \cdot \mathbf{J}^{\phi}$. This is done in Fig. 9 for 
mixture fraction. There the scatter plots of $\left(\frac{\partial \phi}{\partial t}\right)_{D}$ versus mixture fraction are plotted at all three time instants mentioned in Fig. 3. The conventional conditional average of the scattered data are superimposed as the red lines in each plot. Three $y$ locations on the upper branch of the flame are frequently examined throughout this paper. They are: the location corresponding to peak mixture fraction fluctuation $\xi_{r m s, \max }$ (hereafter referred to as $y_{P M}$ ), the locations corresponding to $75 \%$ of $\xi_{r m s, \max }$ on the lean (referred to as $y_{P L}$ ) and rich (referred to as $y_{P R}$ ) sides. The $y$ locations corresponding to $y_{P M}, y_{P L}$, and $y_{P R}$ change with time as the flame develops. Only locations $y_{P M}$ and $y_{P L}$ are shown in Fig. 9.

The conditional diffusion data points are less scattered with increasing time. Except in Fig. 9 (b), the standard deviations based on all the data at one location are on the order of ten times the mean values. The mean values are not negligible compared to the standard deviations, which violates the usual high-Reynolds-number assumptions. In Fig. 9 (b), the standard deviation is 1000 times larger than the mean value. This is because this location is outside the flame, and there the Reynolds number is relatively higher than inside the flame zone, due to the lower viscosity. It can be seen that the test case is not perfect for testing mixing models under high-Reynolds-number conditions. However, the current database provides the best data that are currently available. For a modeling study under similar combustion and flow conditions, the mean drift term $\mathcal{J}^{\phi}$ should be considered explicitly to accurately model this flame.

\subsection{Specification of model constants}

Initial values for model constants $a, b$, and $c$ were established based on passive scalar results in the statistically stationary, homogeneous, isotropic, constant-mean-scalar-gradient test case in Sec. III of [21]; those values are listed in Table 2. There a normalized scalar variance $V_{\phi}(t)$ was defined as,

$$
V_{\phi}(t) \equiv \frac{\left\langle\phi^{2}\right\rangle}{\left(G \sigma T_{L}\right)^{2}},
$$

where $G$ is the value of the constant mean scalar gradient, $\phi^{\prime}$ is the scalar fluctuation, and $\sigma$ is the r.m.s. velocity. In the limit as $t \rightarrow \infty$ (the stationary state), the normalized scalar variance was found to be approximately equal to $3.3\left(V_{\phi}(\infty) \approx 3.3\right)$. By satisfying the dispersion-consistency condition and applying the statistically stationary state condition [21], the relationship of the SPMM model 
constants and flow statistics was established as follows:

$$
\begin{array}{r}
b=\frac{1}{1+a}, \\
V_{\phi}(\infty)=\frac{1}{c}+\frac{2+a}{a} .
\end{array}
$$

Equation 44 sets a lower limit on $a$, such that $c$ remains positive and finite: $a_{\min }=2 /\left(V_{\phi}-1\right)$. Then for any value of $a$ that satisfies this requirement, the values of $b$ and $c$ follow from Eqs. 43 and 44, respectively. For the baseline case, $a$ was chosen to be unity, so that $b=0.5$ and (for $\left.V_{\phi}(\infty)=3.3\right) c=3.27$.

Due to the different nature of turbulence in the current test flame, it has been observed that the standard value $c=3.27$ over-predicts the mixing rates. To separate the effect of mixing rates from the effect of composition-space localness, $c$ is first estimated by matching the scalar dissipation rate that is implied from SPMM to that implied from DNS. With the errors in $c$ thus minimized for each time and location, the remaining discrepancy shown below in the conditional diffusion is mostly attributed to the description of the localness of the model.

To match the scalar dissipation rates between SPMM and DNS, the governing equation for a passive scalar $\xi$ is employed, as shown in Eq. 45:

$$
\frac{D \xi}{D t}=\frac{1}{\rho} \nabla \cdot\left(\rho \mathcal{D}_{\xi} \nabla \xi\right)
$$

Multiplying both sides of Eq. 45 by $-2 \rho \xi$ and taking conventional average, Eq. 45 becomes

$$
\left\langle-2 \rho \xi \frac{D \xi}{D t}\right\rangle=\left\langle 2 \rho \mathcal{D}_{\xi} \nabla \xi \cdot \nabla \xi\right\rangle-\left\langle\rho \mathcal{D}_{\xi} \nabla^{2} \xi^{2}\right\rangle-\left\langle\nabla \xi^{2} \cdot \nabla\left(\rho \mathcal{D}_{\xi}\right)\right\rangle
$$

It has been confirmed using the DNS database that the second and third terms on the right-hand side of Eq. 46 are negligible compared to the first term, and the difference between $\left\langle 2 \rho \mathcal{D}_{\xi} \nabla \xi \cdot \nabla \xi\right\rangle$ and $\left\langle 2 \rho \mathcal{D}_{\xi} \nabla \xi^{\prime \prime} \cdot \nabla \xi^{\prime \prime}\right\rangle$ is indiscernible. Thus, $\left\langle-2 \rho \xi \frac{D \xi}{D t}\right\rangle /\langle\rho\rangle$ is a good representation of the scalar dissipation rate based on mixture fraction. Using this idea, in the baseline case, $c$ is obtained using Eq. 47 ,

$$
\left\langle\rho(\mathbf{x}, t) \phi(\mathbf{x}, t)\left(\frac{\partial \phi}{\partial t}\right)_{D N S}\right\rangle=\left\langle\rho(\mathbf{x}, t) \phi(\mathbf{x}, t)\left(\frac{d \phi}{d t}\right)_{S P M M}\right\rangle
$$

where $\left(\frac{\partial \phi}{\partial t}\right)_{D N S}$ and $\left(\frac{d \phi}{d t}\right)_{S P M M}$ are defined in Eqs. 32 and 33, respectively. Model constant $c$ is embedded in Eq. 33, and thus can be determined using Eq. 47. The model constant $c$ that is determined this way is denoted $c(o p t)$. 
Similar methods are applied in the evaluation of the IECM model in this paper, to avoid the complications from improper choices of mixing rates.

For a stand-alone transported PDF simulation, a possible algorithm to determine the values of $c$ on the fly is to employ the relationship in Eq. 44. Because none of the original assumptions (homogeneous, isotropic, statistically stationary) holds at each step of the temporal jet simulation, especially during early stages of the flame development, $V_{\phi}(\infty)$ is replaced by $V_{\phi}(t)$, which renders Eq. 44 as an approximation. Then $V_{\phi}(t)$ is calculated according to Eq. 42 , where the constant gradient $G$ is replaced by the local gradient $G^{*}(y)=\frac{\partial\langle\xi\rangle}{\partial y}$. Once $a$ is chosen, the corresponding model constant $c$ (denoted as $\left.c\left(V_{\phi}\right)\right)$ can be calculated according to Eq. 44. It is of interest to compare $c($ opt $)$ and $c\left(V_{\phi}\right)$, and this is discussed in Sec. 4.5.

\subsection{Extraction of conditional diffusion from DNS}

It is straightforward to extract the conditional diffusion for both the IECM and IEM models, because all of the conditioning variables in Eqs. 38-41 are deterministic in DNS. Simple binning is employed to calculate the conditional means. It is noted that the standardized Gaussian variables involved in both methods result in different sets of $R^{*}$ for different realizations. However, the difference in $R^{*}$ does not change the statistical results if enough samples are used to obtain them, and it has been confirmed that the statistics extracted using different sets of standardized Gaussian variables are identical.

For this statistically one-dimensional transient flame, at time $t$ and transverse location $y$, there are $n_{x} \times n_{z}$ grid points in the homogeneous $x$ and $z$ directions that can be used to compute statistics. For the $n$th point, the mass fraction or mixture fraction $\phi^{(n)}$, density $\rho^{(n)}$, molecular diffusivity $\mathcal{D}_{\phi}^{(n)}$, and molecular diffusion $\left(\frac{\partial \phi}{\partial t}\right)_{D N S}^{(n)}$ are calculated. Here $R^{(n)}$ and $S^{(n)}$ are retrieved from previous calculations. The sample space $(\psi)$ for the scalar $\phi$ is partitioned into $J$ bins, and the sample-space $R$ corresponding to shadow displacement $R^{*}$ is partitioned into $K$ bins. The DNS estimate of the conditional diffusion at the bin centers $\left(\psi_{j+1 / 2}\right.$ and $\left.R_{k+1 / 2}\right)$ is then obtained by a conventional double-conditioning technique. Similarly for the SPMM, $\left(\frac{d \phi}{d t}\right)_{S P M M}^{(n)}$ is evaluated for grid point $n$, and the same double-conditioning technique is applied.

A parametric study on bin size was conducted (not shown), and it was found that $J=K=50$ bins is adequate in most cases. At certain locations (fuel core or coflow), the bins near the minimum and maximum edges occasionally suffer from reduced sample sizes. Since most of the evaluations 
are not at those locations, 50 bins are used in all the subsequent results. Uniform bins are used for composition variables (and for velocity, in the case of IECM), where the minimum and maximum values over the computational domain are taken as the upper and lower limits.

A similar approach is followed for the IECM model. An example of the conditional diffusion extracted from DNS for the IECM model $\left(D^{I E C M}\right)$ and extracted according to the IECM model $\left(D_{M}^{I E C M}\right)$ is shown in Fig. 10; similar figures for SPMM are shown and discussed in Sec. 4. Here the mixing rate (i.e., the value of $c_{U}$ ) is determined by matching the scalar dissipation rate between DNS and the IECM model, as described in Sec. 3.5. There the one-point, one-time normalized (by $\left.1 / t_{j}\right)$ conditional diffusion of mixture fraction is shown as a function of mixture fraction $(\xi)$ and the normalized transverse instantaneous velocity $v / v_{r m s}$ at $t_{M E}$ at $y_{P M}$.

To interpret Fig. 10, one can imagine that each point with coordinate $\left(v_{i}, \xi_{j}\right)$ is associated with a fluid particle whose instantaneous velocity is $v_{i}$ and whose mixture fraction is $\xi_{j}$. The conditional diffusion at point $\left(v_{i}, \xi_{j}\right)$ is essentially the diffusion source term which controls the evolution of the particle's mixture fraction due to molecular transport. As a passive scalar, the mixture fraction $\xi_{j}$ of the fluid particle increases due to positive conditional diffusion, and $\xi_{j}$ decreases due to negative conditional diffusion. Figure 10 (a) shows positive diffusion in the low- $\xi$ regions (bottom half), and negative diffusion in the high- $\xi$ region. This means that fluid particles with high fuel content (high- $\xi$ ) tend to have their mixture fraction reduced due to the negative conditional diffusion, so that such fluid particles mix with leaner mixtures. The conditional diffusion in Fig. 10 (a) is also a function of $v$, with higher $|v|$ being associated with higher absolute values of the conditional diffusion. Figure 11 shows a scatter plot of $y$-direction velocity versus mixture fraction $\xi$ at the same time and location. It can be seen that large velocity magnitude (greater than $2 v_{r m s}$ ) is associated with the fuel-rich side of mixture fraction, which explains the large conditional diffusion seen on the left and right edges of the contour plot in Fig. 10 (a). The conditional diffusion extracted from the IECM model (Fig. 10(b)) also shows that there is a negative diffusion associated with fuel particles (high mixture fraction) and there is a positive diffusion associated with the oxidizer (low mixture fraction). However, two distinct differences between Fig. 10 (a) and Fig. 10 (b) can be observed. First, the locations of zero conditional diffusion (white band) are somewhat different. For example, the IECM model shows larger variation of the zero conditional diffusion band with velocity for low velocity magnitudes, which suggests that IECM over-emphasizes the role of velocity on molecular mixing in that region. A second difference is that the IECM model does not capture 
the high-conditional-diffusion region for high velocity magnitudes, especially on the negative (high $\xi)$ side. It can be seen that the conditional diffusion does depend on velocity, but the nature of this dependency might not be as simple as that implied by the IECM model.

The difference between the DNS conditional diffusion and the conditional diffusion corresponding to a particular mixing model can be used as a quantitative metric of model performance. A simple global metric is the normalized residual defined as:

$$
\hat{r}_{\text {res }}=\frac{\sum_{i=1}^{J \times K}\left(D_{i}^{M}-D_{i}\right)^{2}}{\sum_{i=1}^{J K} D_{i}^{2}}
$$

where $D_{i}^{M}$ denotes the conditional diffusion calculated from the model for a bin $i$ in $R^{*}-\xi$ space (or $v-\xi$ space for IECM model), while $D_{i}$ denotes the conditional diffusion extracted from DNS for the same bin. For example, the normalized residual for the IECM model at the same time and location as shown in Fig. 10 is 2.13, which implies that the r.m.s. of the error is approximately $146 \%$.

The normalized residuals are quantified and discussed further in Sec. 4.2.

\section{Results and discussion}

The evolution of shadow displacement is discussed first, by examining iso-contours of shadow displacement and the correlations between shadow displacement and velocity and mixture fraction. In the next two subsections, the conditional diffusion of mixture fraction from DNS is compared with that from SPMM using Methods 1 and 2, respectively. Following that, the conditional diffusion

of individual species are discussed. Variations in the values of model constants $c$ and $a$ are discussed in the next two subsections.

\subsection{Shadow displacement}

Snapshots of instantaneous contours of the shadow displacement on the central $z=0$ plane are shown in Fig. 12, at the three instants in time. Here Method 1 (ODE integration) has been used, with the baseline values of the model constants $a$ and $b$ (Table 2). The stoichiometric mixture fraction line is superimposed on each plot, to indicate the flame locations. The flame core and the coflow are separated (approximately) by the stoichiometric-mixture-fraction line. The values of $R^{*}$ approach zero in the coflow region. Between the stoichiometric lines, fine-scale structure can be seen in $R^{*}$. Figure 3 shows that local extinction begins at approximately $4.5 t_{j}$, and that the flame is essentially fully reignited by approximately $15 t_{j}$. The shape of the stoichiometric line changes 
during the local-extinction-reignition process, indicating a possible transition from a flame-folding mechanism to edge-flame propagation [37, 38]. Premixed flame fronts have also been identified during the re-ignition stage for a similar configuration with ethylene fuel [39].

By construction, $R^{*}$ is correlated with the transverse velocity $v$ and with the mixture fraction $\xi$; this can be seen in Fig. 13, where profiles of the correlation coefficients between $R^{*}$ and $\xi\left(\rho_{R^{*}} \xi\right)$ and between $R^{*}$ and $v\left(\rho_{v R^{*}}\right)$ as functions of $y$ are plotted at different instants in time. Results for two different values of model constant $a(a=1$ and $a=10)$ are shown, with $b=\frac{1}{a+1}$ (the value of $c$ does not affect $R^{*}$ in a priori studies). Two dashed boxes whose edges cut through the $50 \%$ $\xi_{r m s, \max }$ locations are plotted to indicate the positions of the reaction zones. Only the regions within the turbulent flame core $\left(\xi_{r m s}>0.1 \xi_{r m s, \max }\right)$ are of interest.

It can be seen that $R^{*}$ and $v$ are always negatively correlated, while the correlation between $R^{*}$ and $\xi$ can be either positive or negative; $\rho_{v \xi}$ and $\rho_{R^{\star} \xi}$ have opposite signs at all locations. Mixture fraction is better correlated with $R^{*}$ than with $v$. A strong correlation between shadow displacement and mixture fraction implies localness in composition space during mixing with SPMM, which is a key desirable attribute (Table 1). Here $\left|\rho_{R^{*} \xi}\right|$ is higher than $\left|\rho_{v \xi}\right|$, whereas an even larger difference was reported in [21]. This is a consequence of the limitations of performing a priori tests for SPMM, the complexity of this flame, and/or inappropriate values for the model constants.

With increasing $a$, the absolute value of peak in $\rho_{R^{*} \xi}$ decreases, while the absolute value of $\rho_{v R^{*}}$ increases. In general, increasing the value of $a$ decreases the localness of SPMM in composition space. For $a=10, R^{*}$ and $v$ are almost perfectly (negatively) correlated. This is consistent with the observation made in [21], that SPMM essentially reduces to IECM in the limit $a \rightarrow \infty$. This is explored further in Sec. 4.6.

The magnitudes of the correlation coefficients do not change significantly with time. From this, one can conclude that the local-extinction and reignition processes do not affect the degree of correlation between $R^{*}$ and $v$ and between $R^{*}$ and $\xi$. This supports the surrogate approach (Method 2), which relies on these correlation coefficients.

To compare the localness captured by Method 1 and Method 2, the correlation coefficients between $R^{*}$ and $\xi$ are plotted at each $y$ location at $t_{R I}$ in Fig. 14. In general, the values of the correlation coefficients calculated for both methods are quite similar. The peak correlation coefficient from Method 2 is slightly higher than that from Method 1. Close to the central $y$ plane, the correlation coefficients from Method 1 are slightly higher than those from Method 2. Neither 
method shows a correlation coefficient of 0.9 or above at any locations.

\subsection{Conditional diffusion: Method 1 (ODEs for $R$ and $S$ )}

The conditional diffusion calculated directly from DNS (Eq. 34) and that calculated according to the SPMM formulation (Eq. 35, using Method 1, baseline values of the model constants $a, b$, and $c$ from Eq. 47) are compared for mixture fraction at the three time instants labeled in Fig. 3. Figures 15, 16 and 17 show the conditional diffusion of mixture fraction from DNS and SPMM at PM, PL, and PR at the three time instants.

Similar to the IECM example in Sec. 3.6, the conditional diffusion is normalized by the jet frequency $\left(1 / t_{j}\right)$, and $R^{*}$ is normalized by the r.m.s. of $R^{*}\left(R_{r m s}^{*}\right)$ at each $y$ location. For all time instants, it has been found that $R_{r m s}^{*}$ is approximately half of the corresponding Taylor microlength scale at the corresponding $y$ location. This gives an indication of the size of the region in physical space from which the shadow displacement effectively draws information for the mixing model.

The conditional diffusion of mixture fraction extracted from SPMM is qualitatively similar in structure to that extracted from DNS (e.g., positive versus negative regions, zero line). SPMM gives zero conditional diffusion where the scalar is equal to its corresponding conditional mean value. DNS gives zero conditional diffusion where it actually is zero according to its definition. It is desirable that these two zero-bands are located at the same locations in $R^{*}-\xi$ space. It can be seen that SPMM and DNS have zero conditional diffusion at similar locations in $R^{*}-\xi$ space, but not at exactly the same locations. A larger discrepancy is shown in the comparison between the IECM model and DNS shown in Fig. 10. Similar behavior has also been observed in comparing IEM and DNS results [40] (there the zero conditional diffusion location is a point). The location of the zero conditional diffusion line can be controlled by changing the values of the model constants that determine the shadow displacement. Another possible improvement is to build in information about intermittency.

The similarities between the conditional diffusion contours from DNS and from SPMM are higher at $y_{P M}$ (Fig. 15) and $y_{P L}$ (Fig. 16) at all time instants than at $y_{P R}$ (Fig. 17). It is speculated that the intermittency effects might play a role at $y_{P R}$ where fuel particles have higher probabilities to be surrounded by other fuel particles, which results in a zero molecular diffusion at a higher mixture fraction value, as seen in Fig. 17. SPMM captures the position of the zero-lines and the 
locations of peak conditional diffusion values remarkably well at several locations (e.g., $t_{M E}$ and $y_{P M}, t_{R I}$ and $\left.y_{P M}\right)$. Many highly-localized structures are observed in the conditional diffusion from DNS. For example, the conditional diffusion changes signs three times in Fig. 16 (b) along the left boundary of the contours from $\xi=1$ to $\xi=0.4$. These highly-localized structures are not captured by the SPMM model at the same time and location. The formulation of SPMM dictates that its prediction is fairly organized (i.e., there is a conditional mean line, and negative and positive conditional diffusion above and below that line). As a model, SPMM is ultimately employed in a RANS or LES context, where the grid size does not capture the smallest localized structures in any case.

Besides the locations of the zero-conditional-diffusion bands, results from SPMM also show better prediction of the locations of the maximum and minimum conditional diffusion, compared to the IECM model at same time and location (Fig. 10). As discussed in Sec. 3.6, conditional diffusion does depend on velocity; however, this dependency can be further improved by adding scalar history information that is manifested through $R^{*}$.

By integrating the conditional diffusion over the scalar space (mixture fraction, in this case) and $R^{*}$, one can recover the unconditional mean diffusion, which should be equal to zero by definition and construction (property I in Table 1). For both DNS and SPMM, it has been confirmed that the unconditional diffusion is very close to zero; small deviations result from the fact that the Reynolds-number of the flow is moderate, as discussed in Sec. 3.4.

The global metric $\hat{r}_{\text {res }}$ is also examined to evaluate the overall agreement between DNS and SPMM, at $t_{M E}$ and $t_{R I}$, at all three locations. Results are summarized in Table 3 . The first two columns of Table 3 are obtained for Method 1. Column 1 contains results that are calculated using the values of $c$ described in Eq. $47(c(o p t))$, while Column 2 contains results that are calculated based on values of $c$ calculated using Eq. $44\left(c\left(V_{\phi}\right)\right)$. Similarly, Columns 3 and 4 contain results based on Method 2, using $c(o p t)$ and $c\left(V_{\phi}\right)$, which are discussed further in Sec. 4.3. As a comparison between different mixing models, results calculated for the IECM model are also listed in Table 3. Column 5 is calculated according to Eq. 47, using the definitions of IECM model (Eq. 36). The values of $c$ in Column $6(c(\phi))$ is obtained by

$$
c(\phi)=\frac{\tau}{\tau_{\phi}},
$$

with $\tau=k / \varepsilon$ and $\tau_{\phi}=\frac{\overline{\xi^{\prime \prime 2}}}{\left\langle 2 \mathcal{D}_{\xi} \nabla \xi^{\prime \prime} \nabla \xi^{\prime \prime}\right\rangle_{\rho}}$. 
The normalized residuals calculated using $c($ opt $)$ are smaller than those calculated using $c\left(V_{\phi}\right)$, but they are quite close to one another. For both time instances, the normalized residuals are smaller at $y_{P R}$ than at $y_{P M}$ and $y_{P L}$. Values that are larger than unity are observed at $y_{P M}$ and $y_{P L}$, which are not desirable. The normalized residuals obtained from SPMM using Method 1 are smaller than those obtained using the IECM model.

In summary, in Method $1, R^{*}$ is forced to be highly correlated with mixture fraction by means of the variable $X$ (recall Sec. 3.2), and the model constant $c$ is extracted from scalar dissipation rates of mixture fraction. In Sec. 4.4, the conditional diffusions of $\mathrm{CO}$ and $\mathrm{H}_{2} \mathrm{O}$ are examined using $R^{*}$ and $c$ obtained from this section. The comparison there can be used to further evaluate the performance of SPMM in terms of preserving localness and predicting mixing rates for each individual species.

\subsection{Conditional diffusion: Method 2 (surrogate for shadow displacement)}

Figure 18 shows the normalized conditional diffusion of mixture fraction, based on the normalized surrogate $R_{s g}^{*}$ (Method 2), at $y_{P M}$ and $y_{P R}$ at $t_{R I}$. It can be observed that the range of $R_{s g}^{*} / R_{r m s}^{*}$ is similar to that of $R_{O D E}^{*} / R_{r m s}^{*}$ shown in Figs. 15, 16 and 17, but they are not exactly the same.

The comparison between the DNS and modeled conditional diffusion iso-contours is similar to that shown in Sec. 4.2, with fewer fine structures revealed. The results from DNS and SPMM (Method 2) are not visually as similar as those from Method 1, but the normalized residuals are consistently smaller than those from Method 1 (Table 3, Columns 3 and 4). Fewer spots of extreme values of conditional diffusion are observed in the DNS results shown in Fig. 18. This might contribute to the smaller residual values observed in Table 3. Another possible source of improvement might come from the slightly higher correlation of $R_{s g}^{*}$ with $\xi$ in Method 2 as shown in Fig. 14. However, it has to be kept in mind that the normalized residual is only one global parameter; it is not advisable to draw definitive conclusions from one global quantity. Both Method 1 and Method 2 involve surrogates of the shadow displacement. It is difficult to conclude which method provides a better representation of what the shadow displacement might look like in a stand-alone calculation. A transported PDF simulation should be carried out to further verify the phenomena observed in this study.

Computationally, the extraction of $R_{s g}^{*}$ is approximately 100 times less expensive than obtaining 
$R_{O D E}^{*}$ using Method 1, because Method 1 requires integration of ODEs in time.

\subsection{Conditional diffusion of individual species}

In this study, the shadow displacement is forced to be closely correlated with mixture fraction. It has been demonstrated that correlating the shadow displacement with mixture fraction enforces a level of localness in the composition space (Secs. 4.2 and 4.3). Here, the $R^{*}$ generated in Sec. 4.2 and the model constant $c$ calculated using Eq. 47 are used to compare the conditional diffusion of individual species extracted from DNS and from the SPMM formulation. In this way, the localness of SPMM in terms of species molecular mixing can be directly evaluated. Figures 19 and 20 show the conditional diffusion comparisons for $\mathrm{CO}$ and $\mathrm{H}_{2} \mathrm{O}$ at $t_{R I}$ at $y_{P M}$ and $y_{P R}$, respectively. Generally speaking, SPMM can capture the shapes of the conditional diffusion in composition space for $\mathrm{CO}$ and $\mathrm{H}_{2} \mathrm{O}$ quite well. The model can even capture some of the small local structures. For example, the sign changes of condition diffusion of $\mathrm{H}_{2} \mathrm{O}$ near $Y_{H 2 O} / Y_{H 2 O, r m s}=3, R^{*} / R_{r m s}^{*}=3$ are well captured by the model. Similar details can also be observed in the conditional diffusion plot for CO. Both iso-contour plots show darker color than the DNS plots, which suggests an over-prediction of mixing rates based on the mixture-fraction-based scalar dissipation rates.

The mixing rates based on the scalar dissipation rates of each species are calculated, and the implied $c$ constants are plotted in Fig. 21 at $t_{R I}$. The same Lagrangian time scale $T_{L, 2}$ is used to calculate $c$ in all cases. Interestingly, the mixing rates of major species $\mathrm{H}_{2} \mathrm{O}$ and $\mathrm{CO}$ are quite similar inside the flame core. The ratios of the values of $c$ obtained from mixture fraction and the values obtained from $\mathrm{CO}$ or $\mathrm{H}_{2} \mathrm{O}$ are approximately between 1.5 and 2.5. It is also observed (not shown) that the mixing rates of minor species (e.g., $\mathrm{OH}$ and $\mathrm{H}$ ) are highly influenced by differential diffusion and the premixed flame features that exist in the flame core after re-ignition [38], which is out of the scope of the current study. For those species, the corresponding values of $c$ are usually higher than that of mixture-fraction-based $c$.

\subsection{Variations in model constant $c$}

The baseline values of $c$ used in Sec. 4.2 were extracted by matching scalar dissipation rates at each $y$-location (Eq. 47). It is observed that the values of $c$ obtained in this way are much smaller than the standard model constant derived in [21]. This difference might be due to the highly-idealized flow conditions in [21]. 
The values of $c$ that are derived from Eq. $47(c(o p t))$ are compared with the values of $c$ extracted from Eq. $44\left(c\left(V_{\phi}\right)\right)$ in Fig. 22 (a) to (c) for different time instants. $c\left(V_{\phi}\right)$ ranges between 0.2 and 0.4 in the flame zones, which is consistently smaller than $c(o p t)$. The normalized residuals calculated using $c($ opt $)$ are slightly larger than those calculated using $c\left(V_{\phi}\right)$ (Table 3), indicating a slightly better global prediction by using $c(o p t)$. However, the difference is not dramatic.

Since $c\left(V_{\phi}\right)$ is obtained through $V_{\phi}$, it is of interest to examine $V_{\phi}$. The profile of $V_{\phi}$ is compared with that of $\rho_{V \xi}^{-2}$ in Fig. 22 (d) for $t_{R I}$. Only the central region of the flame where turbulence is intense and the mixture is reactive is plotted. It can be seen that the value of $V_{\phi}$ approaches its corresponding stationary-state value (3.3) in the flame zones with increasing time. At all locations and time instants, $\rho_{v \xi}^{-2}$ is a good approximation to $V_{\phi}$. Thus, the modified model coefficient $c$ can be written as a function of $\rho_{v \xi}$. Values of $\rho_{v \xi}$ can be readily extracted on-the-fly for a velocitycomposition PDF method, which can then be used to form a dynamic model constant $c$ for SPMM.

\subsection{Variations in model constant a}

As mentioned in Sec. 1, SPMM is related to other mixing models including IEM, IECM and MMC. It has been shown in Sec. 4.1 that the value of $a$ controls the localness of the model. By changing $a$, SPMM can approach the behavior of other mixing models. For example, as $a$ approaches $\infty, b=\frac{1}{1+a} \rightarrow 0$ and $c=c_{\text {min }}$. In this limit, $R^{*}$ and $v$ are perfectly negatively correlated (Fig. 13), so that conditioning on $R^{*}$ is identical to conditioning on $v$, and therefore SPMM degenerates to the IECM model.

To further illustrate this point, results obtained with $a=1$ and $a=10$ for SPMM from DNS $\left(D^{S P M M}\right)$ are shown in Fig. 23, for $t_{M E}$ at $y_{P M}$. All sub-figures in Figs. 23 are generated directly from the DNS database using Eqs. 34 and 38; i.e., we are not assessing model accuracy here. Because of the negative correlation between $R^{*}$ and $v$, the conditional diffusion distribution should be mirrored to judge the similarity between Fig. 23 (b) and 23 (c). The SPMM and IECM results are more similar to one another for $a=10$ compared to $a=1$. For $a=10$, the shapes of the outlines of iso-contours, the locations of the maximum and minimum conditional diffusion, and the corresponding magnitudes are almost perfectly mirrored between SPMM and IECM. It is possible that $a=10$ is sufficiently large for the present test flame. It has been observed that results from the SPMM model are quite sensitive to the value of $a$; in [21], values of $a$ at 1.15 and 1.2 were shown to give very different results. A recent a posteriori study [22] shows that by adjusting $a$ and 
$c$, the SPMM can show behaviors similar to those of EMST or IEM in terms of scatter plots of temperature. The choices of $a$ and $c$ play an important role when using SPMM.

\section{Conclusions}

The newly-proposed shadow-position mixing model (SPMM) has been examined, using a recently generated DNS database for a temporally evolving DME jet flame. Compared to alternative models, SPMM possesses more of the attributes desired in a mixing model (Table 1). Earlier work on SPMM [21] has been limited to highly idealized canonical systems. Here its behavior in a more complex turbulent flame is studied. The test flame is a temporally evolving, statistically one-dimensional slot-jet DME flame at a moderate jet Reynolds number of 13,050 [24]. The DNS features a reduced 30-species DME chemical mechanism, and the flame parameters were chosen to ensure that the flame undergoes significant local extinction and re-ignition. The inhomogeneous, non-stationary nature of the configuration including strong local extinction and reignition pose a significant challenge to SPMM, or to any other mixing model.

A keystone for SPMM is the use of a conditioning variable called the shadow displacement (denoted $R^{*}$ ), which is a constructed stochastic variable. By design, $R^{*}$ is correlated with mixture fraction and with velocity, so that conditioning on $R^{*}$ is similar to conditioning on one or both of these key physical quantities. DNS provides all the necessary statistics required to construct $R^{*}$ (with some additional assumptions), so that an a priori study can be carried out to understand the behavior and evaluate the performance of SPMM. One caveat of an a priori study of SPMM is that the mechanism of correlating $R^{*}$ and mixture fraction is missing, and that correlation has to be enforced by applying the SPMM model. Here two different methods (denoted as Methods 1 and 2) are proposed to extract $R^{\star}$ from the DNS solution, with varying levels of approximation. In Method 1, one reconstructs statistics of $R^{*}$ by solving ODEs for its mean $(R)$ and variance $(S)$. Then, the maximum possible correlation with mixture fraction is built into $R^{*}$. In Method 2, one calculates a surrogate of $R^{*}$ using local flow statistics. Method 1 requires fewer approximations, but it is more difficult to apply. Method 2 is of interest for its ease of implementation and low computational cost.

Flow statistics show that the flow field remains highly nonstationary at the end of the simulation,

and the Reynolds-stress profiles show that the flow field is highly anisotropic. Smooth profiles of the turbulent diffusivity and Lagrangian time scales are prerequisites for evaluating the SPMM 
model. Here the turbulent diffusivity and Lagrangian time scales extracted from DNS are smoothed using cross-validation and other techniques. Quantitative comparisons between DNS and SPMM are based largely on the conditional diffusion of mixture fraction and of individual species. The definition and quantification of the conditional diffusion from DNS and from different mixing models is a nontrivial task, and a proposed formulation is presented in this paper. The conditional diffusions of mixture fraction from both methods are analyzed and compared, and most of the subsequent analysis is based on Method 1 .

By comparing the correlation coefficients among shadow displacement, velocity and mixture fraction extracted using Method 1, it is shown that the shadow position is more strongly correlated with composition than with velocity. The correlation coefficient between shadow displacement and mixture fraction is higher than that between velocity and mixture fraction. The strong correlation between shadow displacement and scalars ensures the realization of localness in SPMM. However, due to the limitation of the a priori study, a perfect correlation between the shadow displacement and composition can not be achieved.

Conditional diffusion evaluated from DNS and from SPMM (Method 1) have been compared, and good qualitative agreement was found. To rule out the influence of inaccuracies in mixing rate predictions, the model parameter $c$ was obtained by matching the scalar dissipation rates of mixture fraction locally in time and space. Better predictions of the zero conditional diffusion lines, and maximal and minimal conditional diffusion are observed from SPMM, compared to the IECM model. The global normalized residuals obtained from SPMM are consistently smaller than those obtained from the IECM model.

Similar comparisons were made based on Method 2. The global normalized residuals obtained from Method 2 are consistently smaller than those obtained from Method 1. However, the isocontours of conditional diffusion are similar to those for Method 1, and some local details are missing from the iso-contours of conditional diffusion extracted using Method 2. Compared to the complex procedure of obtaining $R^{*}$ from Method 1 , Method 2 is a viable alternative to evaluate the SPMM in other statistically 1-D configurations in the future.

Suitable values for model constants $c$ and $a$ also were explored in this study. It was found that a value of the model constant $c$ that is calculated from the normalized scalar flux is quite close to the value that is calculated based on matching the scalar dissipation rates. The relationship between SPMM and IECM was studied by changing the model parameter $a$, which controls the correlation 
between $R^{*}$ and velocity. In this test flame, increasing $a$ from 1 to 10 effectively reduces SPMM to IECM.

The test flame used here is not an ideal test case for testing SPMM in the high-Reynolds number limit, because of its moderate Reynolds number (non-negligible mean drift of molecular diffusion) in this flame. Stand-alone transported PDF simulations at higher Reynolds numbers are needed to further evaluate the performance of the model.

\section{Acknowledgments}

This research is supported by the Combustion Energy Frontier Research Center (CEFRC), an Energy Frontier Research Center funded by the U.S. Department of Energy (DOE), Office of Science, Office of Basic Energy Sciences (BES) under Award No. DE-SC0001198. Sandia is a multiprogram laboratory operated by Sandia Corporation, a Lockheed Martin Company, for the United States Department of Energy under contract DE-AC04-94AL85000. Computer allocations were awarded by the Department of Energy's INCITE award at the Oak Ridge Leadership Computing Facility (OLCF) at the Oak Ridge National Laboratories (ORNL).

\section{Appendices}

\section{A. Derivation of statistically stationary solution for $R$ and $S$}

The statistically stationary solutions for $R$ and $S$ can be obtained by considering the nature of the evolution equation for the shadow displacement $R^{*}$. A good approximation of the stationarystate solution $\left(R_{s s}\right)$ is,

$$
R_{s s}=\left\langle R \mid v^{\prime \prime}\right\rangle_{\rho}
$$

Because $R^{*}$ and $v^{\prime \prime}$ have a joint-normal distribution, $\left\langle R \mid v^{\prime \prime}\right\rangle_{\rho}[30]$ can be shown to be:

$$
\left\langle R \mid v^{\prime \prime}\right\rangle_{\rho}=\langle R\rangle_{\rho}+v^{\prime \prime} \frac{\left\langle R v^{\prime \prime}\right\rangle_{\rho}}{\left\langle v^{\prime \prime 2}\right\rangle_{\rho}}
$$

and $v^{\prime \prime}$ can be modeled using a Langevin equation as:

$$
d v^{\prime \prime}=-\frac{v^{\prime \prime}}{T_{L}} d t+\left(2 \sigma^{2} / T_{L}\right)^{1 / 2} d W .
$$


Here, $W$ is the Wiener process associated with the fluid particle motion. From Eqs. 15 and 52, it follows that,

$$
\begin{gathered}
\langle R\rangle_{\rho}=\left\langle v^{\prime \prime}\right\rangle_{\rho}=0, \text { and } \\
\left\langle R v^{\prime \prime}\right\rangle_{\rho}=-\frac{\sigma^{2} T_{L}}{1+a} .
\end{gathered}
$$

Thus $R_{s s}^{*}$ can be written as:

$$
R_{s s}=\left\langle R \mid v^{\prime \prime}\right\rangle_{\rho}=-\frac{v^{\prime \prime} T_{L}}{1+a},
$$

and $S_{s s}$ can be obtained by setting the left-hand side of Eq. 16 to zero. The resulting initial conditions at time $t_{0}$ are then: where the instantaneous $y$-component fluctuating velocity $v^{\prime \prime}$ is taken from the DNS, and the turbulent diffusivity and time scale are extracted as explained earlier. Time $t_{0}$ is taken to be approximately $5 t_{j}$ in the DNS simulation (recall that the first time instant for analysis is $6 t_{j}$ ), to allow sufficient time for the DNS to purge any potential effects from the somewhat arbitrary initial conditions. 
[1] D. W. Meyer, P. Jenny, Micromixing models for turbulent flows, J. Comput. Phys. 228 (2009) 1275 1293.

[2] R. L. Curl, Dispersed phase mixing: I. Theory and effects in simple reactors, AIChE J. 9 (1963) $175-181$.

[3] J. Janicka, W. Kolbe, W. Kollmann, Closure of the transport equation for the probability density function of turbulent scalar fields, J. Non-Equilib. Thermodyn. 4 (1979) 47-66.

[4] J. Villermaux, J. C. Devillon, Représentation de la coalescence et de la redispersion des domaines de ségrégation dans un fluide par un modèle dinteraction phénoménologique, Elsevier, New York, 1972.

[5] C. Dopazo, E. E. O'Brien, An approach to the autoignition of a turbulent mixture, Acta Astronautica 1 (1974) $1239-1266$.

[6] R. O. Fox, On the relationship between stochastic lagrangian models of turbulence and second-moment closures, Phys. Fluids 6 (1994) 973 - 985.

[7] R. O. Fox, On velocity-conditioned scalar mixing in homogeneous turbulence, Phys. Fluids 8 (1996) $2678-2691$.

[8] S. B. Pope, On the relationship between stochastic lagrangian models of turbulence and second-moment closures, Phys. Fluids 6 (1994) $973-985$.

[9] H. Chen, S. Chen, R. H. Kraichnan, Probability distribution of a stochastically advected scalar field, Phys. Rev. Lett. 63 (1989) 2657.

[10] S. B. Pope, Mapping closures for turbulent mixing and reaction, Theoret. Comput. Fluid Dyn. 2 (1991) $255-270$

[11] A. Y. Klimenko, S. B. Pope, The modeling of turbulent reactive flows based on multiple mapping conditioning, Phys. Fluids 15 (2003) 1907-1925.

[12] A. Y. Klimenko, Matching conditional moments in PDF modeling of nonpremixed combusion, Combust. Flame 143 (2005) 369-385.

[13] S. Subramaniam, S. B. Pope, A mixing model for turbulent reactive flows based on Euclidean minimum spanning trees, Combust. Flame 115 (1998) 487-514.

[14] R. O. Fox, The Fokker-Planck closure for turbulent molecular mixing: Passive scalars, Phys. Fluids A 4 (1992) $1230-1244$. 
[15] R. O. Fox, Improved Fokker-Planck model for the joint scalar, scalar gradient PDF, Phys. Fluids 6 (1994) $334-348$.

[16] D. W. Meyer, P. Jenny, A mixing model for turbulent flows based on parameterized scalar profiles, Phys. Fluids 18 (2006) 035105.

[17] D. W. Meyer, P. Jenny, A mixing model providing joint statistics of scalar and scalar dissipation rate, Proc. Combust. Inst. 32 (2009) $1613-1620$.

[18] M. Hegetschweiler, B. T. Zoller, P. Jenny, Reactive parametrized scalar profiles (R-PSP) mixing model for partially premixed combustion, Combust. Flame 159 (2012) $734-747$.

[19] R. O. Fox, Computational models for turbulent reactive flows, Cambridge University Press, 2003.

[20] D. C. Haworth, Progress in probability density function methods for turbulent reacting flows, Prog. Energy Combust. Sci. 36 (2010) 168-259.

[21] S. B. Pope, A model for turbulent mixing based on shadow-position conditioning, Phys. Fluids 25 (110803) (2013) 1-27.

[22] J. C. K. Tang, A. Kong, E. R. Hawkes, S. B. Pope, J. H. Chen, Evaluating the shadow position mixing model for transported pdf modeling of non-premixed flames, in: 19th Australasian Fluid Mechanics Conference (2014), paper 26.

[23] M. R. Overholt, S. B. Pope, Direct numerical simulation of a passive scalar with imposed mean gradient in isotropic turbulence, Phys. Fluids 8 (1996) 3128 - 3148.

[24] A. Bhagatwala, T. Lu, H. Shen, J. A. Sutton, J. H. Chen, Numerical and experimental investigation of turbulent DME jet flames, Proc. Combust. Inst. 35 (2015) 1157 - 1166.

[25] E. R. Hawkes, R. Sankaran, J. C. Sutherland, J. H. Chen, Scalar mixing in direct numerical simulations of temporally evolving plane jet flames with skeletal CO/ $\mathrm{H}_{2}$ kinetics, Proc. Combust. Inst. 31 (1) (2007) $1633-1640$.

[26] D. O. Lignell, J. H. Chen, H. A. Schmutz, Effects of Damköhler number on flame extinction and reignition in turbulent non-premixed flames using DNS, Combust. Flame 158 (2011) 949 - 963.

[27] T. Echekki, J. H. Chen, Structure and propagation of methano-air triple flames, Combust. Flame 114 (12) (1998) $231-245$. 
[28] S. Tavoularis, S. Corrsin, Experiments in nearly homogeneous turbulent shear flow with a uniform mean temperature gradient. part 1, J. Fluid Mech. 104 (1981) 311 - 347.

[29] S. Tavoularis, S. Corrsin, Effects of shear on the turbulent diffusivity tensor, Int. J. Heat Mass Transfer 28 (1985) $265-276$.

[30] S. B. Pope, Turbulent flows, Cambridge University Press, Cambridge, UK, 2000.

[31] S. B. Pope, Simple models for turbulent flows, Phys. Fluids 23 (2011) 011301-1 - 011301-20.

[32] S. B. Pope, R. Gadh, Fitting noisy data using cross-validated cubic smoothing splines, Commun. Statist. -Simula. 17 (2) (1998) 349-376.

[33] S. B. Pope, A stochastic lagrangian model for acceleration in turbulent flows, Phys. Fluids 14 (2002) $2360-2375$.

[34] P. K. Yeung, S. B. Pope, An algorithm for tracking fluid particles in numerical simulations of homogeneous turbulence, J. Comput. Phys. 79 (1988) 373-416.

[35] M. J. Cleary, A. Y. Klimenko, A generalized multiple mapping conditioning approach for turbulent combustion, Flow Turbul. Combust. 82 (2009) 477-491.

[36] S. B. Pope, R. Tirunagari, Advances in probability density function methods for turbulent reactive flows, in: 19th Australasian Fluid Mechanics Conference (2014), 2014, paper 15.

[37] E. R. Hawkes, R. Sankaran, J. H. Chen, Extinction and reignition in direct numerical simulations of $\mathrm{CO} / \mathrm{H}_{2}$ temporal plane jet flames, in: 5th US combustion National Meeting, 2007.

[38] A. Bhagatwala, E. R. Hawkes, J. H. Chen, Dynamics of extinction and resignation in DNS of a non premixed turbulent DME jet flame, in: 9th US combustion National Meeting (2015), 2015, paper $1 \mathrm{H} 13$.

[39] C. Li, D. O. Lignell, J. H. Chen, T. Lu, Detection of local extinction and re-ignition in non-premixed ethylene-air flames using chemical explosive mode analysis, in: 9th US combustion National Meeting (2015), paper $1 \mathrm{H} 10$.

[40] G. Brethouwer, F. Nieuwstadt, DNS of mixing and reaction of two species in a turbulent channel flow: a validation of the conditional moment closure, Flow Turbul. Combust. 66 (2001) $209-239$. 
Table 1: Desirable properties of molecular mixing models, and characteristics of five mixing models with respect to these criteria. Five models are compared: the modified Curl's (MCD) model, the interaction by exchange with the mean (IEM) model, the interaction by exchange with the conditional mean (IECM) model, the Euclidean minimum spanning tree (EMST) model, and the shadow position mixing model (SPMM). SPMM satisfies the most desired properties among all the models compared.

\begin{tabular}{|c|c|c|c|c|c|}
\hline Mixing models & MCD & IEM & IECM & EMST & SPMM \\
\hline I. Conservation of means & $\checkmark$ & $\checkmark$ & $\checkmark$ & $\checkmark$ & $\checkmark$ \\
\hline II. Correct decay of variances & $\checkmark$ & $\checkmark$ & $\checkmark$ & $\checkmark$ & $\checkmark$ \\
\hline III. Localness in composition space & - & - & - & $\checkmark$ & $\checkmark$ \\
\hline IV. Bounded in allowable composition space & $\checkmark$ & $\checkmark$ & $\checkmark$ & $\checkmark$ & $\checkmark$ \\
\hline V. Turbulent dispersion consistency & - & - & $\checkmark$ & - & $\checkmark$ \\
\hline VI. Relaxation to Gaussian & - & - & - & - & $\checkmark$ \\
\hline VII. Linearity and independence from other scalars & $\checkmark$ & $\checkmark$ & $\checkmark$ & - & $\checkmark$ \\
\hline VIII. Dependence on length scales of scalar field & - & - & - & - & - \\
\hline IX. Dependence on $R e, S c$, and $D a$ & - & - & - & - & - \\
\hline X. Differential diffusion & - & - & - & - & - \\
\hline
\end{tabular}

Table 2: Baseline model constants for SPMM.

\begin{tabular}{ccc}
\hline$a$ & $b$ & $c$ \\
\hline 1 & 0.5 & 3.27 \\
\hline
\end{tabular}


Table 3: Normalized residuals (Eq. 48) at $t_{M E}$ and $t_{R I}$, at all three locations, for Method 1, Method 2 and the IECM model. For Methods 1 and 2, normalized residuals obtained using $c(o p t)$ (Eq. 47) and $c\left(V_{\phi}\right)$ (Eq. 44) are compared. For the IECM model, normalized residuals obtained using $c(o p t)$ (Eq. 47) and $c(\phi)$ (Eq. 49) are compared.

\begin{tabular}{lllllll}
\hline $\begin{array}{l}\text { Time \& lo- } \\
\text { cation }\end{array}$ & M1, c(opt $)$ & $\mathrm{M} 1, c\left(V_{\phi}\right)$ & $\mathrm{M} 2, c($ opt $)$ & $\mathrm{M} 2, c\left(V_{\phi}\right)$ & $\begin{array}{l}\text { IECM, } \\
c(o p t)\end{array}$ & $\begin{array}{l}\text { IECM } \\
c(\phi)\end{array}$ \\
\hline$t_{M E}, y_{P R}$ & 0.76 & 1.45 & 0.3 & 0.55 & 2.18 & 0.72 \\
\hline$t_{M E}, y_{P M}$ & 2.78 & 3.36 & 0.67 & 0.60 & 3.85 & 2.13 \\
\hline$t_{M E}, y_{P L}$ & 1.52 & 2.60 & 0.9 & 1.50 & 2.56 & 2.18 \\
\hline$t_{R I}, y_{P R}$ & 0.65 & 0.95 & 0.21 & 0.40 & 1.97 & 0.93 \\
\hline$t_{R I}, y_{P M}$ & 1.37 & 2.67 & 0.40 & 0.59 & 3.50 & 1.28 \\
\hline$t_{R I}, y_{P L}$ & 4.30 & 9.35 & 3.40 & 7.01 & 8.06 & 2.19 \\
\hline
\end{tabular}


Figure 1: Relationships among several molecular mixing models. IEM: the interaction by exchange with the mean model; IECM: the interaction by exchange with the conditional mean model; EMST: the Euclidean minimum spanning tree model; MMC: the multiple mapping conditioning model (mixture fraction based); SPMM: the shadow position mixing model. 


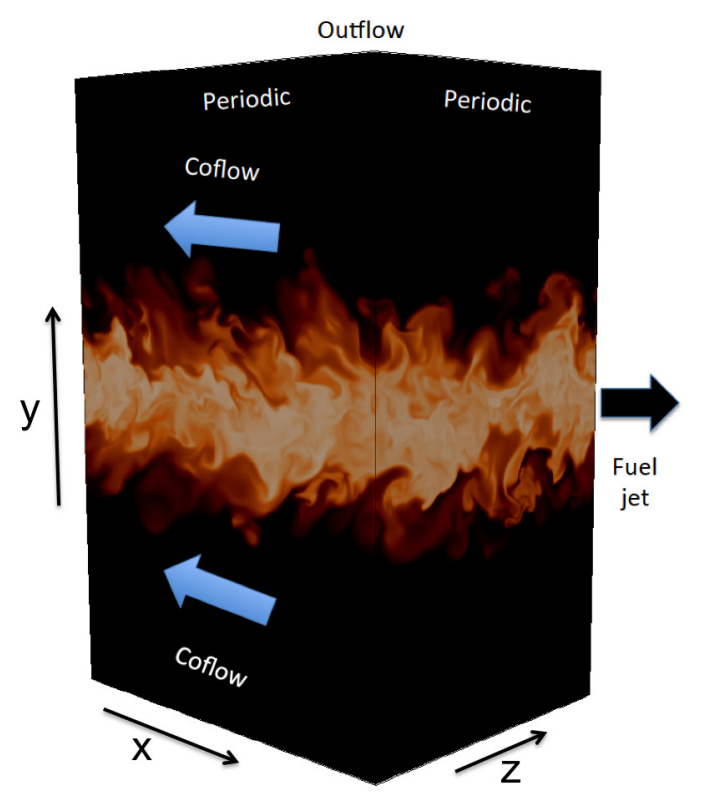

Figure 2: Turbulent DME slot-jet-flame configuration. Color indicates the instantaneous temperature on the outer surfaces of the computational domain. 


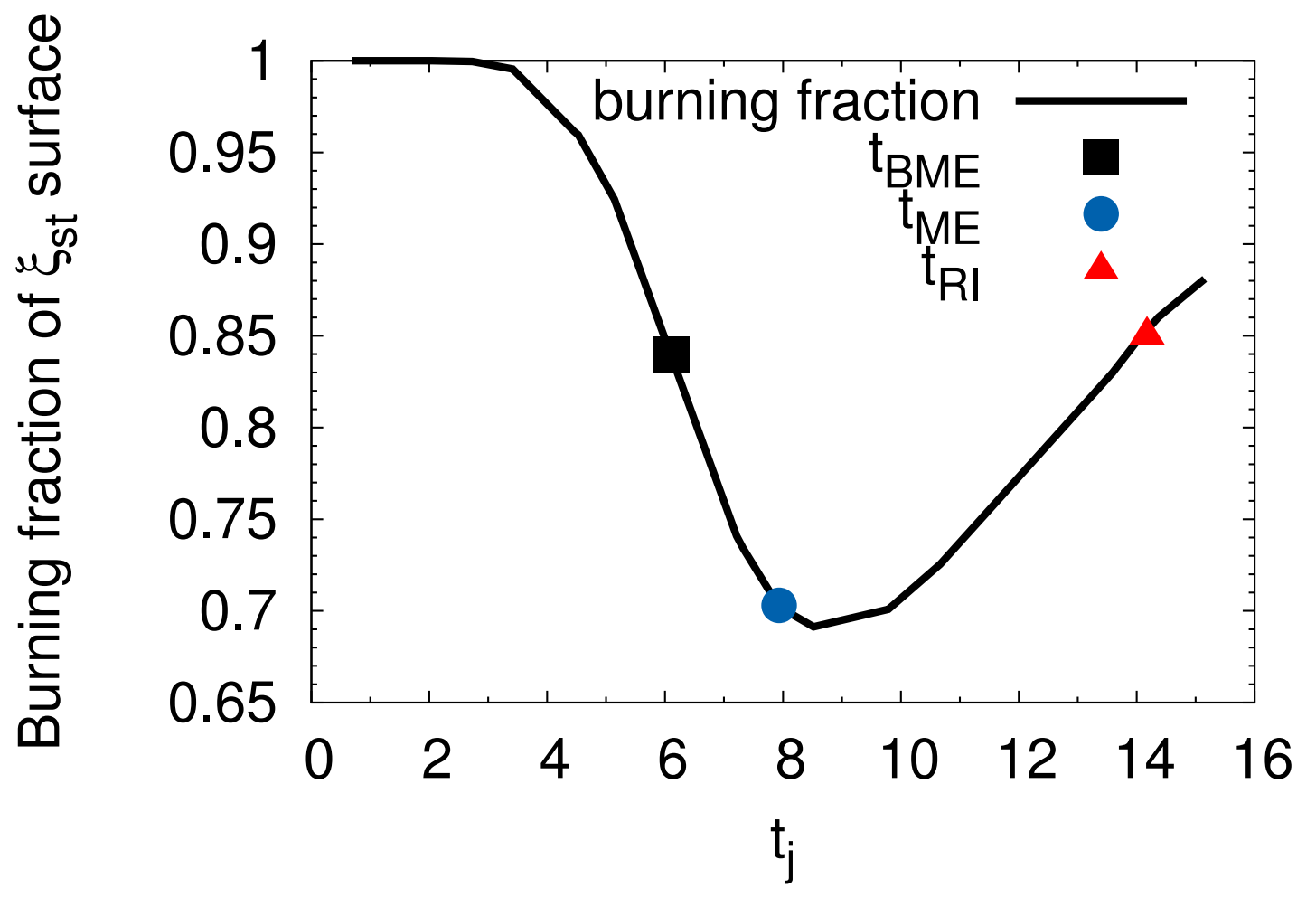

Figure 3: Global burning fraction as a function of time. Symbols indicate the three time instants at which most of the analysis is performed. 


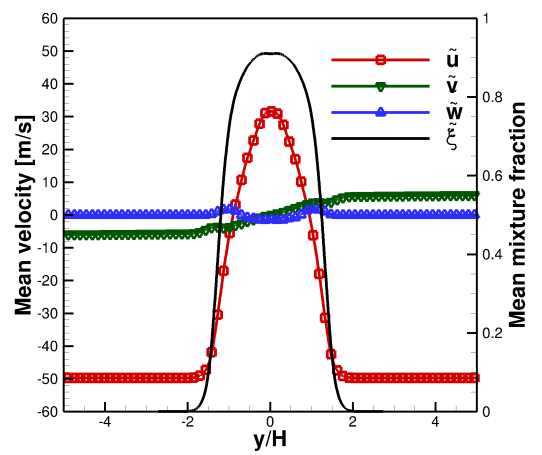

(a) $t_{B M E}$

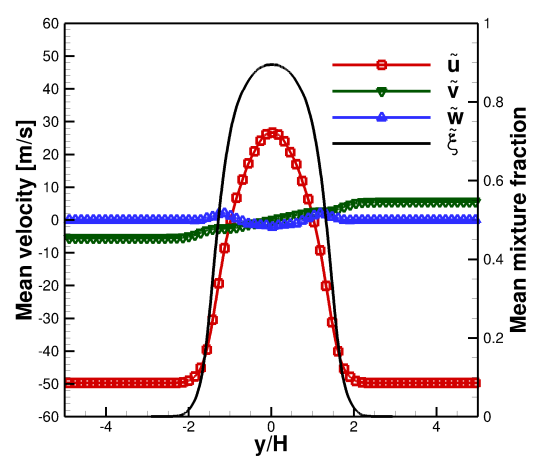

(c) $t_{M E}$

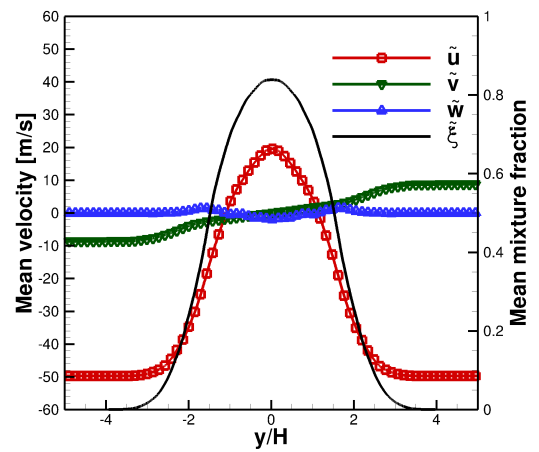

(e) $t_{R I}$

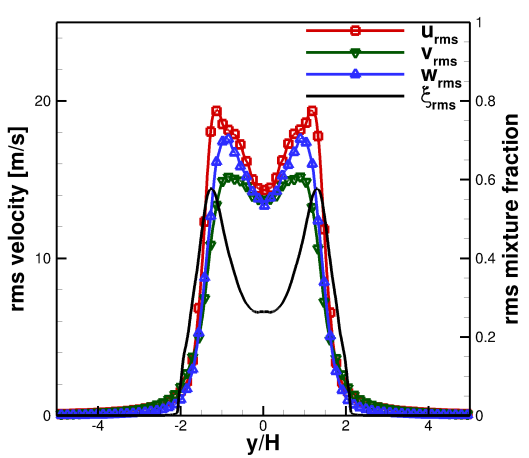

(b) $t_{B M E}$

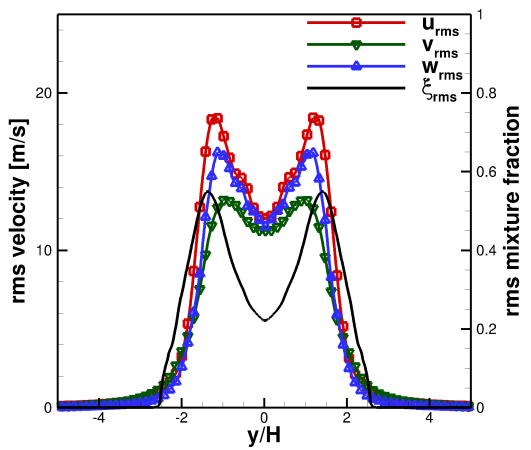

(d) $t_{M E}$

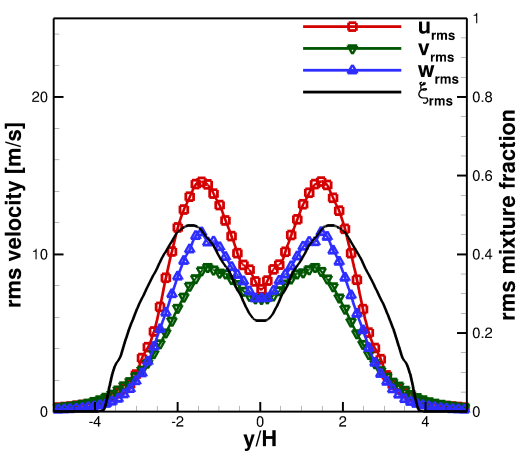

(f) $t_{R I}$

Figure 4: Favre-averaged mean and r.m.s. velocity components and mixture fraction profiles at three time instants. 


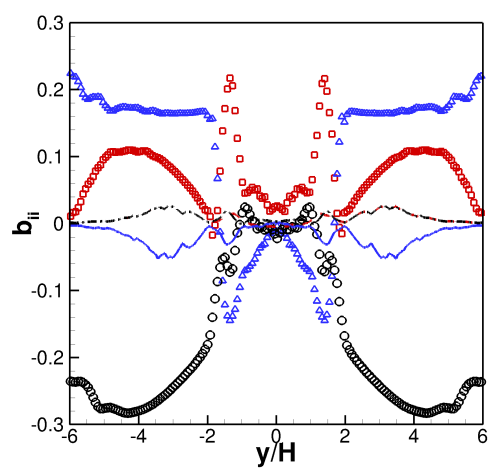

(a) $t_{B M E}$

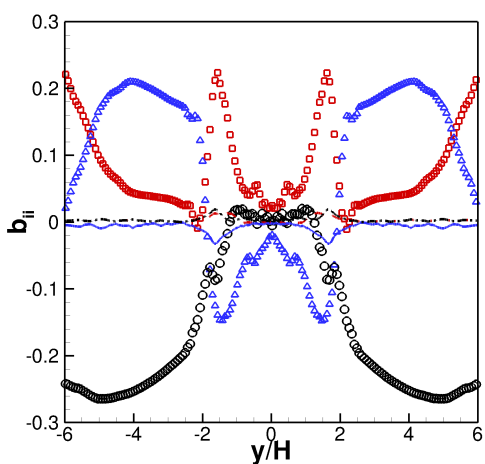

(c) $t_{M E}$

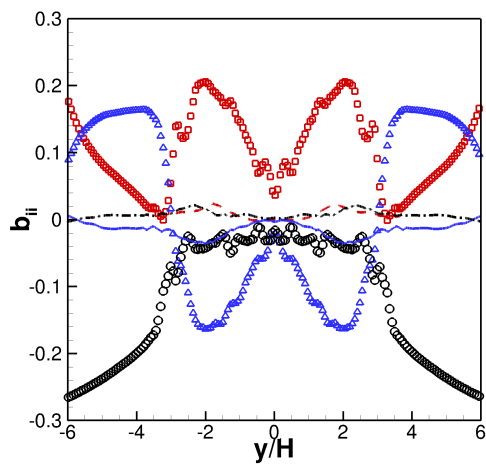

(e) $t_{R I}$

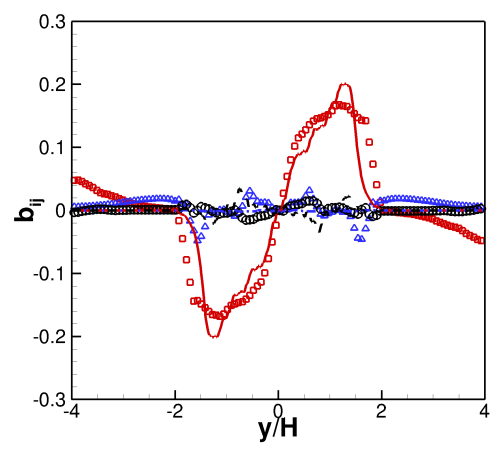

(b) $t_{B M E}$

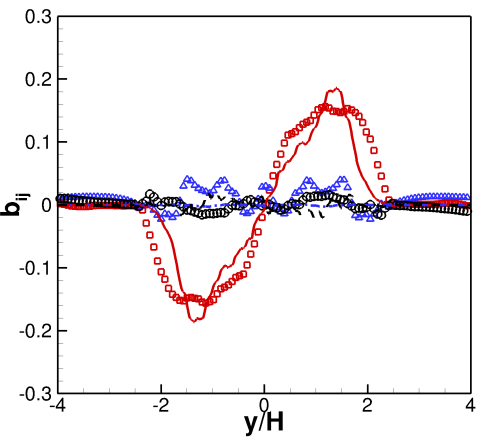

(d) $t_{M E}$

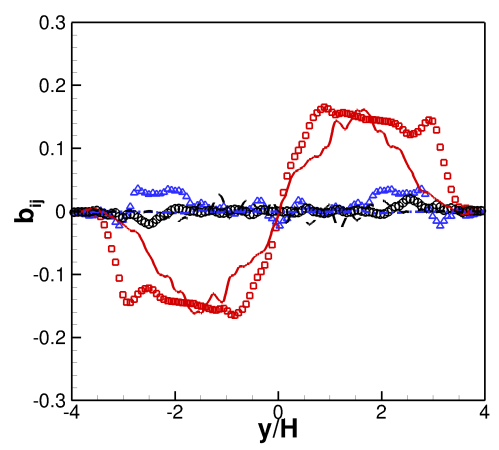

(f) $t_{R I}$
50

51

52

53

54

55

56

57

58

59

60

61

62

63

64

65

Figure 5: Profiles of components of the normalized anisotropy tensor $\left(b_{i j}=\widetilde{u_{i}^{\prime \prime} u_{j}^{\prime \prime}} / \widetilde{u_{k}^{\prime \prime} u_{k}^{\prime \prime}}-\frac{1}{3} \delta_{i j}\right)$ extracted from DNS (symbols) versus those calculated from a $k-\varepsilon$ model (lines) at three time instants. Left column: $b_{11}(\square), b_{22}(\triangle)$, $b_{33}(\circ), b_{11, M}(---), b_{22, M}(-), b_{33, M}(-\cdot--)$. Right column: $b_{12}(\square), b_{13}(\triangle), b_{23}(\circ), b_{12, M}(-), b_{13, M}$ $(-\mathbf{-}), b_{23, M}(-\mathbf{-})$. 


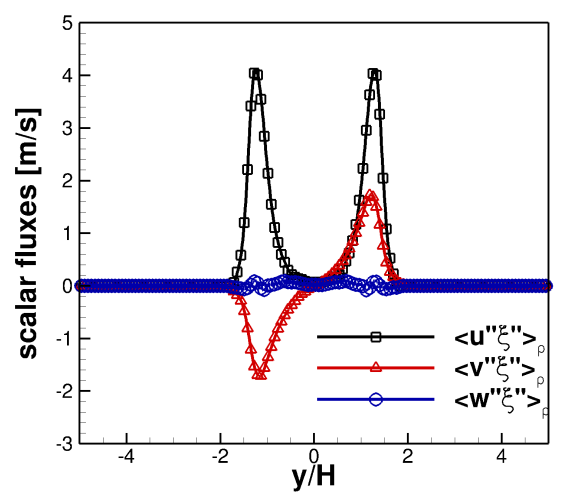

(a) $t_{B M E}$

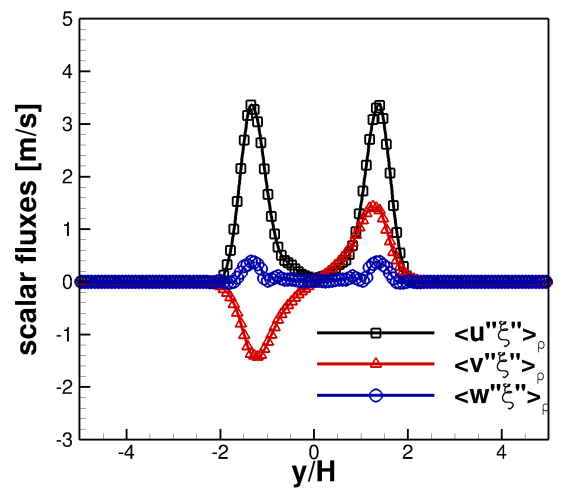

(b) $t_{M E}$

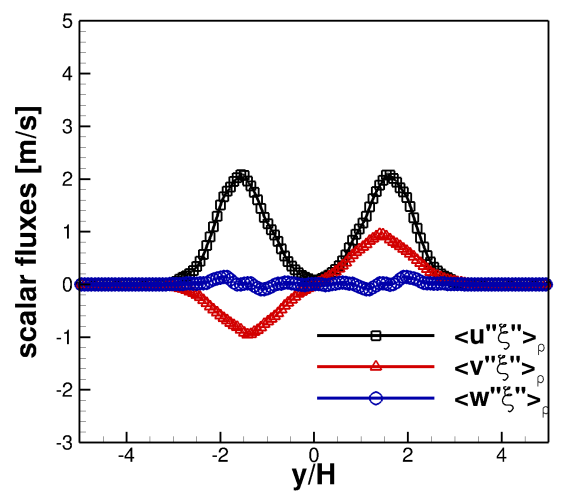

(c) $t_{R I}$

Figure 6: Three components of turbulent mixture fraction flux at three time instants.

61

62

63 


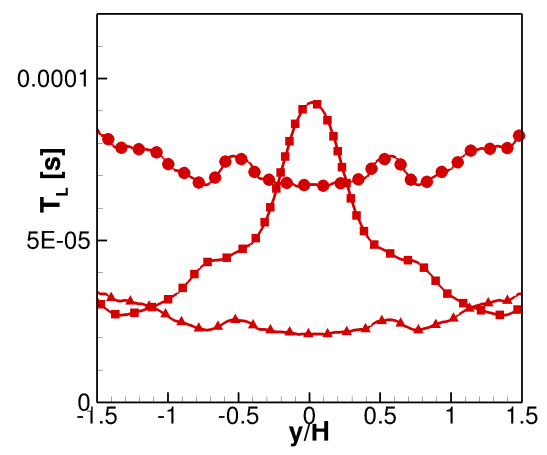

(a) $t_{B M E}$

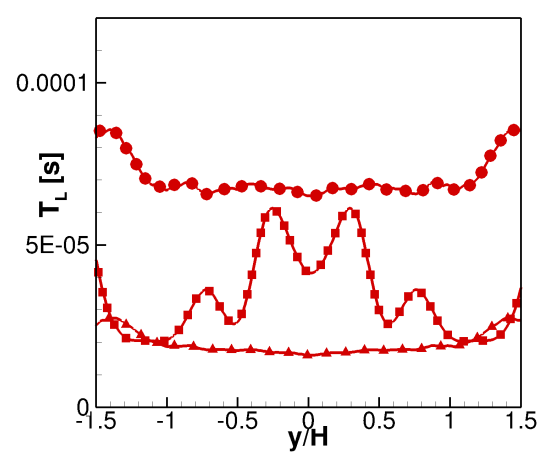

(b) $t_{M E}$

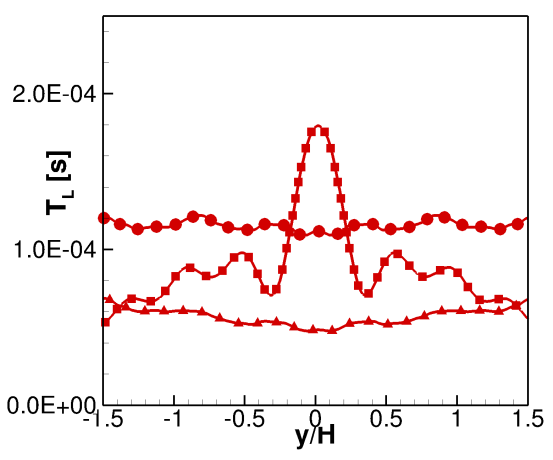

(c) $t_{R I}$

Figure 7: Favre-averaged profiles of $T_{L}$ at three time instants. $T_{L, 1}(-\rightarrow)$ corresponds to the $T_{L}$ calculated using Eq. 9, $T_{L, 2}(-)$ corresponds to the $T_{L}$ calculated using Eq. 10, and $T_{L, 3}(-\bullet)$ corresponds to the $T_{L}$ calculated using Eq. 11. 


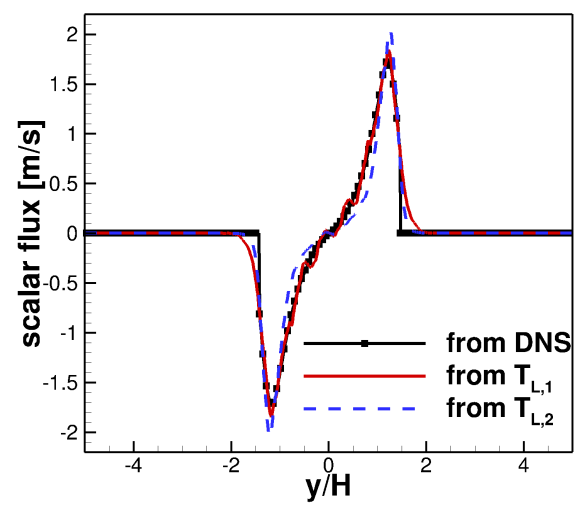

(a) $t_{B M E}$

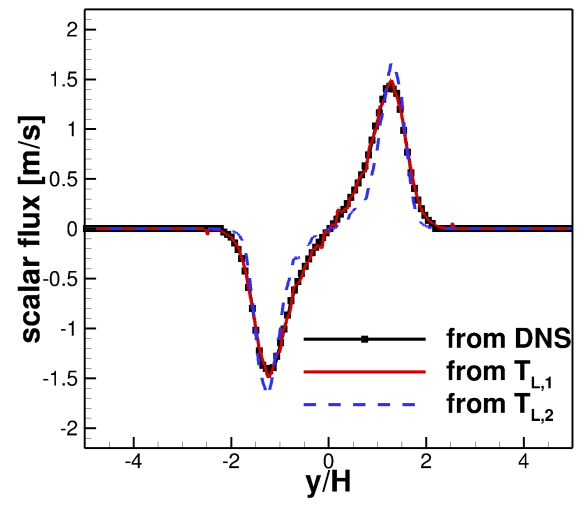

(b) $t_{M E}$

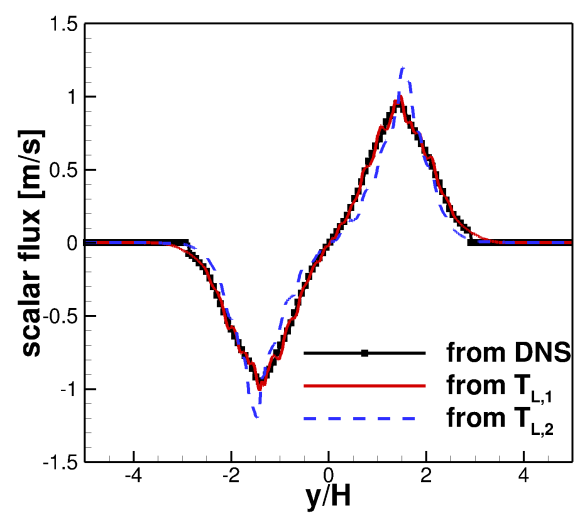

(c) $t_{R I}$

44

Figure 8: Favre-averaged turbulent scalar flux (mixture fraction) profiles calculated directly from DNS and calculated using $T_{L, 1}$ and $T_{L, 2}$ at three time instants. 


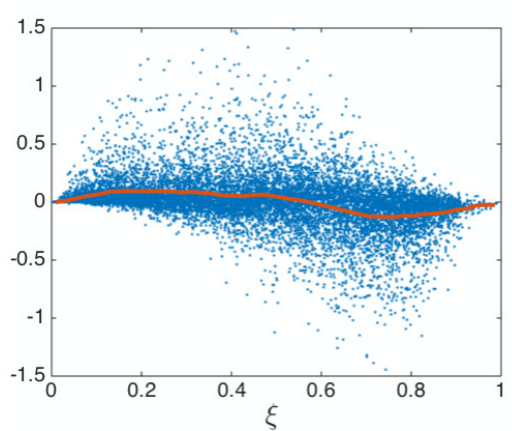

(c) $t_{M E}, y_{P M}$

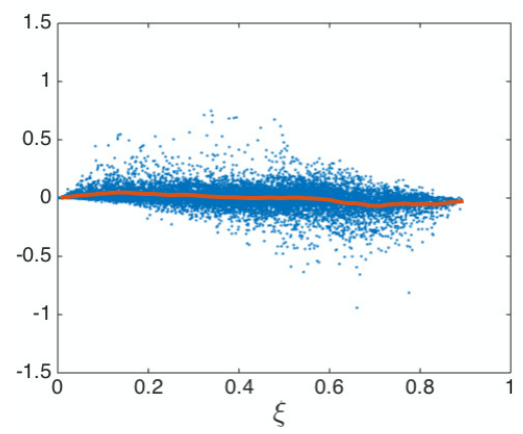

(e) $t_{R I}, y_{P M}$

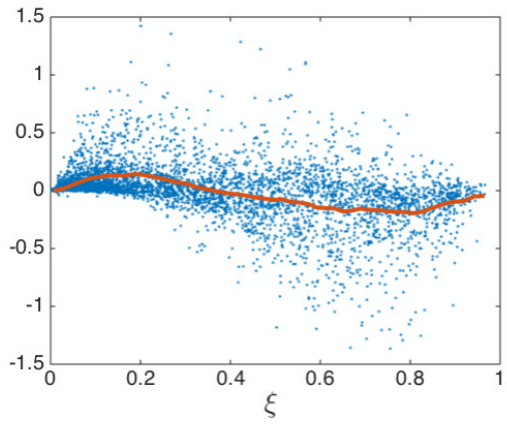

(b) $t_{B M E}, y_{P L}$

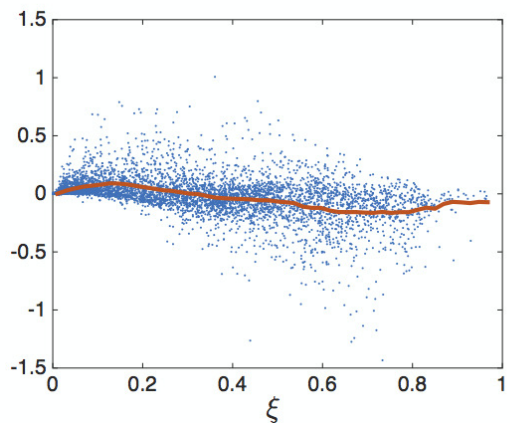

(d) $t_{M E}, y_{P L}$

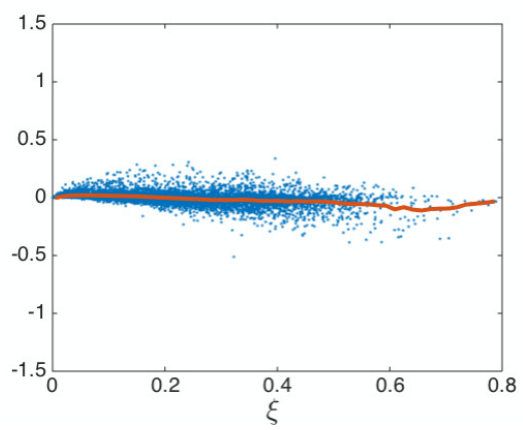

(f) $t_{R I}, y_{P L}$

Figure 9: Scatter plots of $-\frac{1}{\rho} \nabla \cdot \mathbf{J}^{\phi}$ (normalized by $1 / t_{j}$ ) versus mixture fraction $\xi$ at $t_{B M E}, t_{M E}$ and $t_{R I}$ and the two locations $y_{P M}$ and $y_{P L}$. The data points on one $\mathrm{x}-\mathrm{z}$ plane are used, with 50 times downsampling. Red lines are diffusion conditional on mixture fraction. 


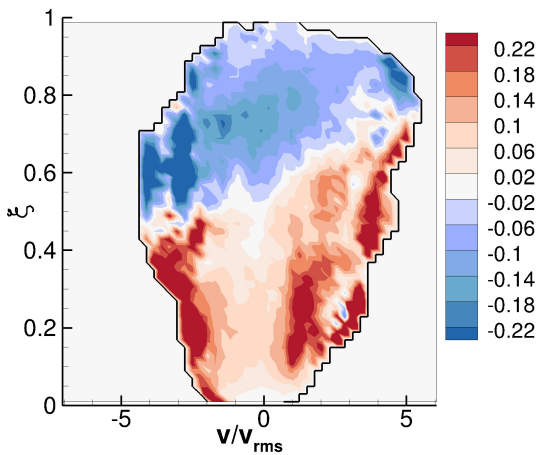

(a) DNS

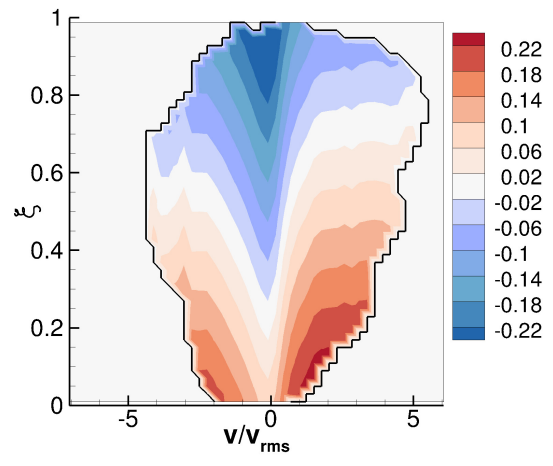

(b) IECM

Figure 10: Normalized conditional diffusion of mixture fraction $\left(D^{I E C M}(\xi) t_{j}\right)$ extracted from DNS and from the IECM model at $t_{M E}$ and $y_{P M}$, where $y_{P M}$ denotes the location of maximum $\xi_{r m s}$.

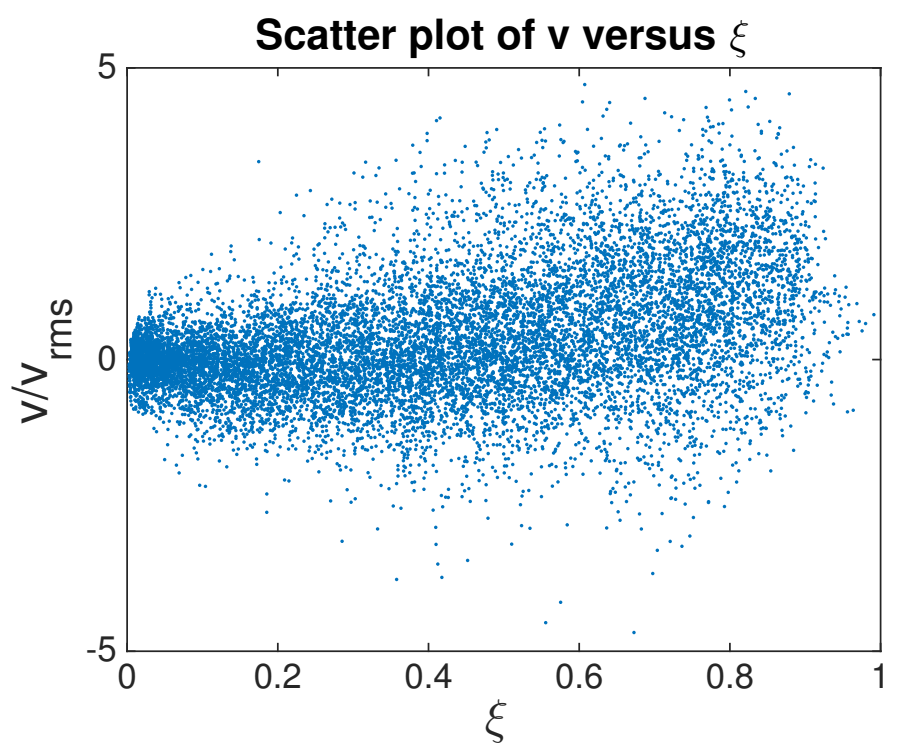

Figure 11: Scatter plot of normalized $y$-direction velocity $\left(v / v_{r m s}\right)$ versus $\xi$ at $t_{M E}$ and $y_{P M}$. 


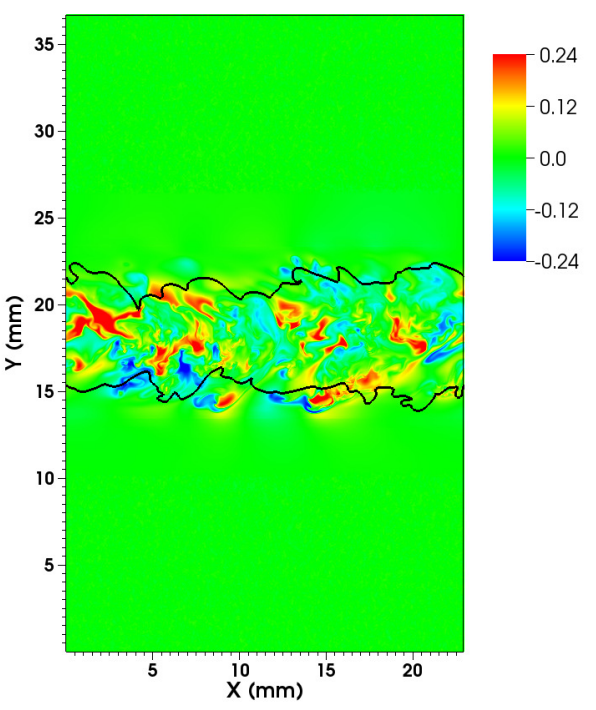

(a) $t_{B M E}$

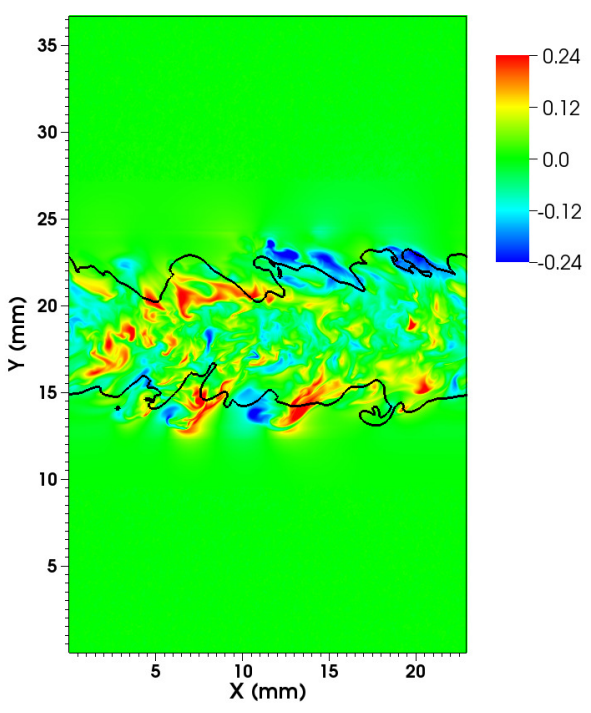

(b) $t_{M E}$
28

29

30

31

32

33

34

35

36

37

38

39

40

41

42

43

44

45

46

47

48

49

50

51

52

53

54

55

56

57

58

59

60

61

62

63

64

65

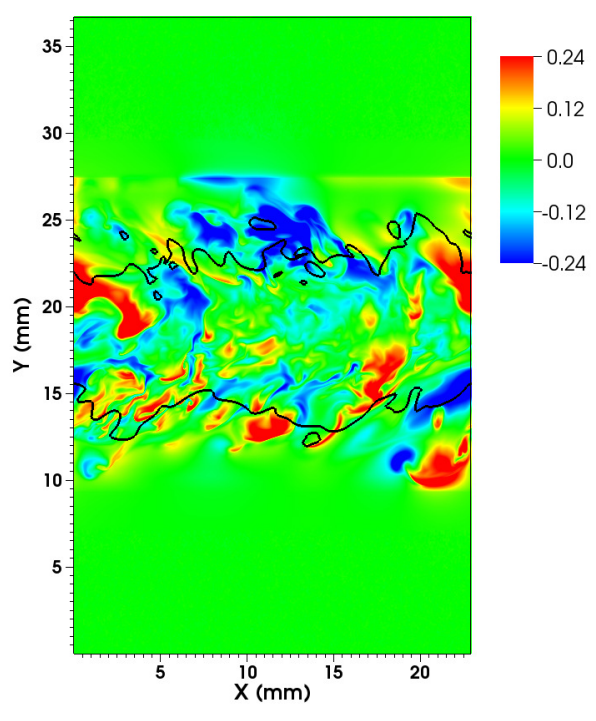

(c) $t_{R I}$

Figure 12: Instantaneous $R_{O D E}^{*}$ fields (normalized by jet width $H$ ) from Method 1 on the central $z$ plane at different times. The black lines correspond to the stoichiometric mixture fraction $\left(\xi_{s t}=0.375\right)$. 


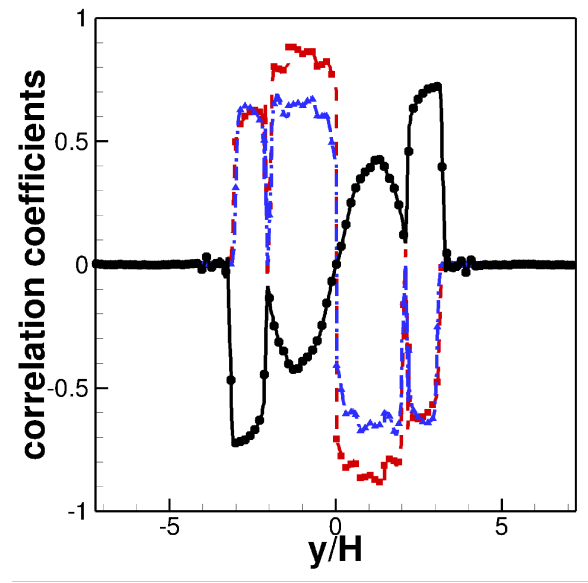

(a) $\rho_{R^{\star} \xi}$ and $\rho_{V \xi}$ at $t_{B M E}$

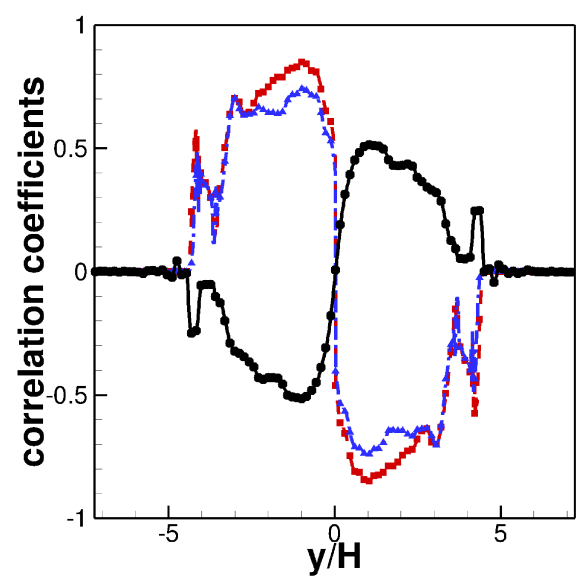

(c) $\rho_{R^{*} \xi}$ and $\rho_{V \xi}$ at $t_{R I}$

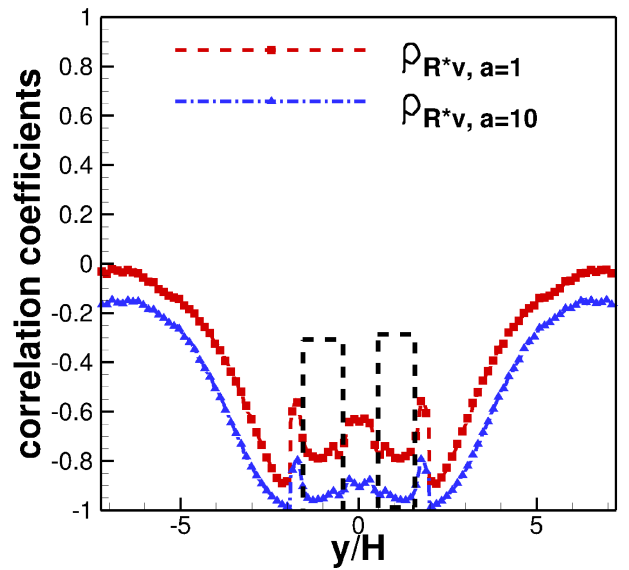

(b) $\rho_{v R^{*}}$ and $\sigma_{\xi}$ at $t_{B M E}$

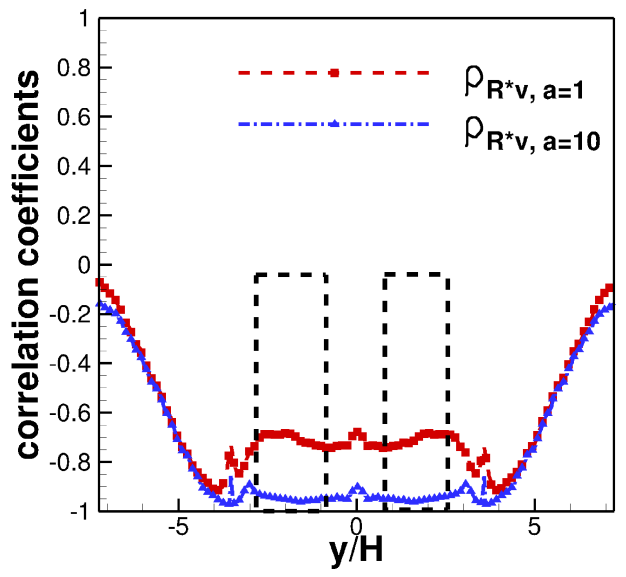

(d) $\rho_{v R^{*}}$ and $\sigma_{\xi}$ at $t_{R I}$

Figure 13: Correlation coefficients between $R_{O D E}^{*}$ (Method 1) and $v$ (right) and between $R_{O D E}^{*}$ and $\xi$ (left) at different time instants. Left column: $\rho_{R^{*} \xi}, a=1(--), \rho_{R^{*} \xi}, a=10\left(-{ }^{-}\right)$, and $\rho_{v \xi}(-\bullet)$. The dashed boxes in (b) and (d) enclose the regions where $\xi_{r m s}$ is larger than $50 \% \xi_{r m s, \text { max }}$ to indicate the flame zones. 


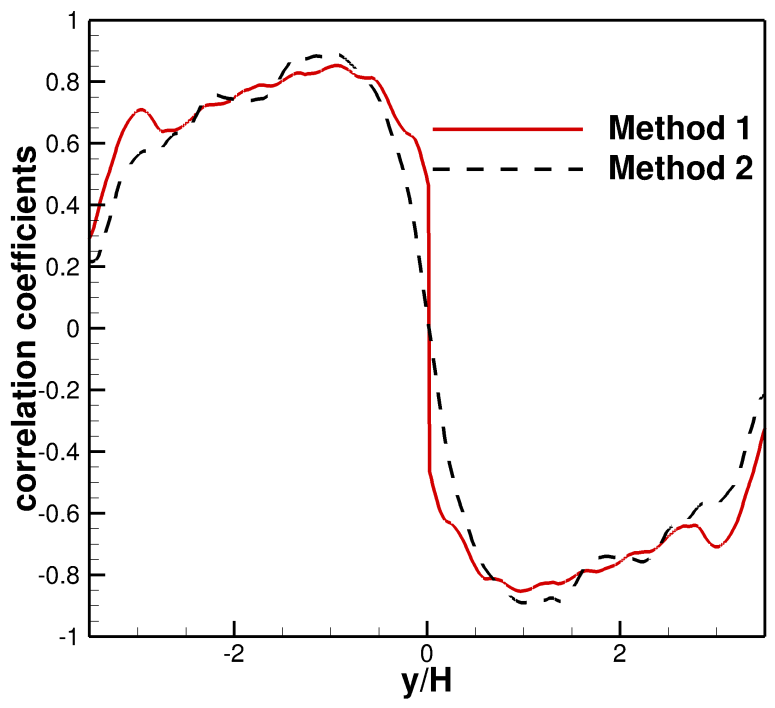

Figure 14: Comparison of correlation coefficients of $R^{*}$ and $\xi\left(\rho_{R^{*} \xi}\right)$ at $t_{R I}$ between Method 1 and Method 2. 


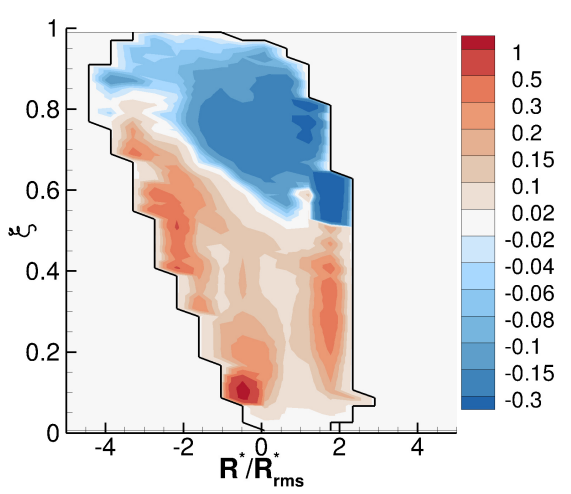

(a) $t_{B M E}, \mathrm{DNS}$

g

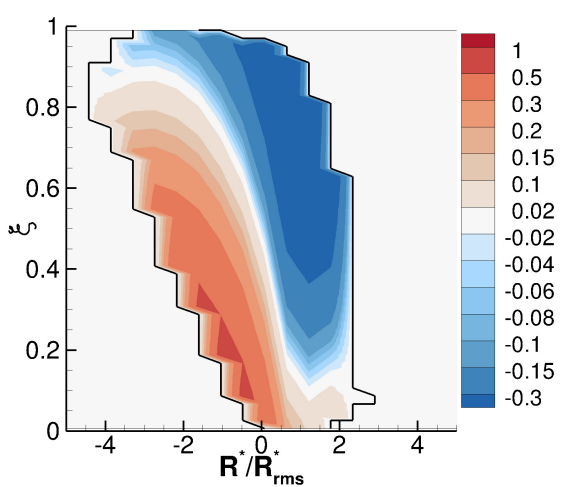

(d) $t_{B M E}, \mathrm{SPMM}$

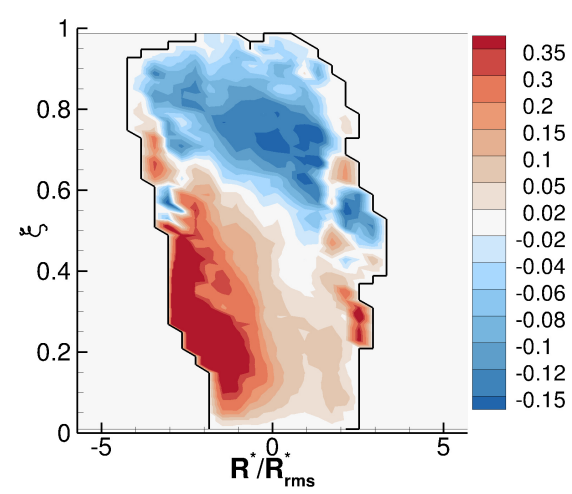

(b) $t_{M E}, \mathrm{DNS}$

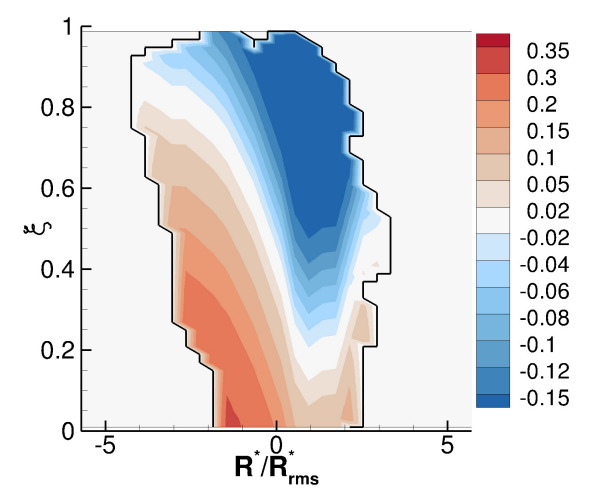

(e) $t_{M E}$, SPMM

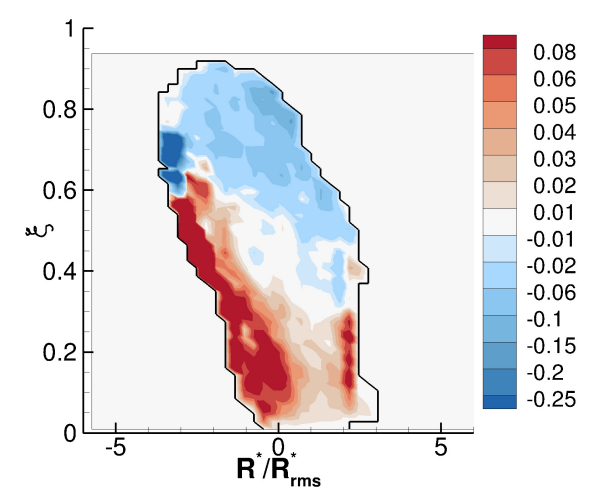

(c) $t_{R I}, \mathrm{DNS}$

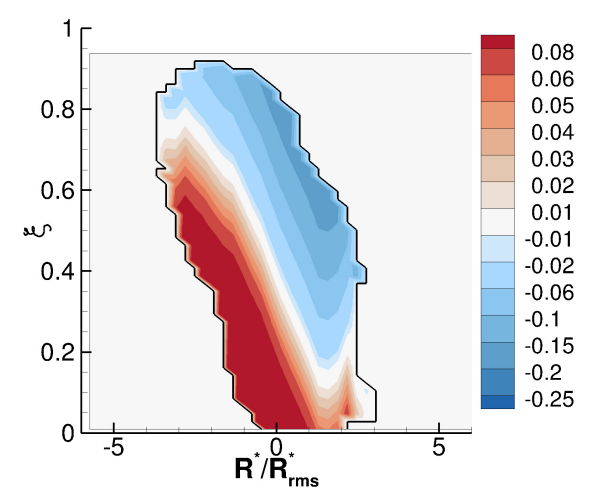

(f) $t_{R I}$, SPMM

Figure 15: Iso-contours of $D^{S P M M}(\xi) t_{j}$ (top row) and versus $D_{M}^{S P M M}(\xi) t_{j}$ (bottom row) at different time instants at $y_{P M}$. $R^{*}$ is normalized by its corresponding r.m.s. at that time and location. 


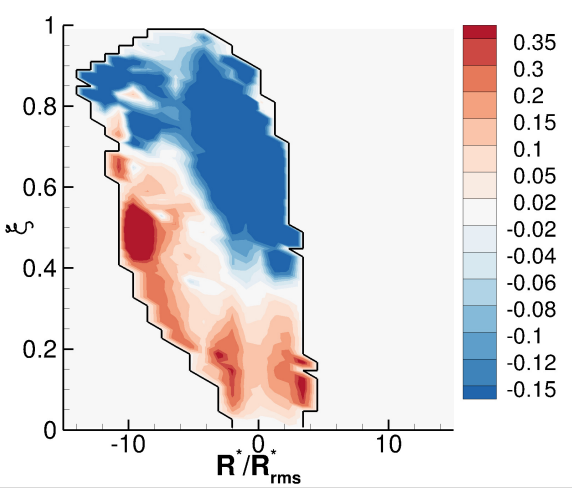

(a) $t_{B M E}$, DNS

cr

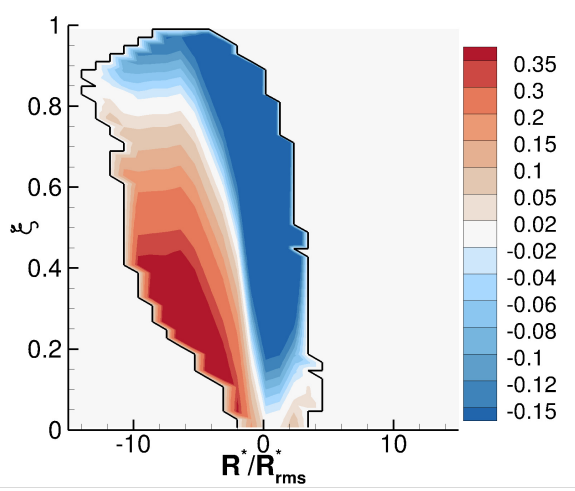

(d) $t_{B M E}$, SPMM

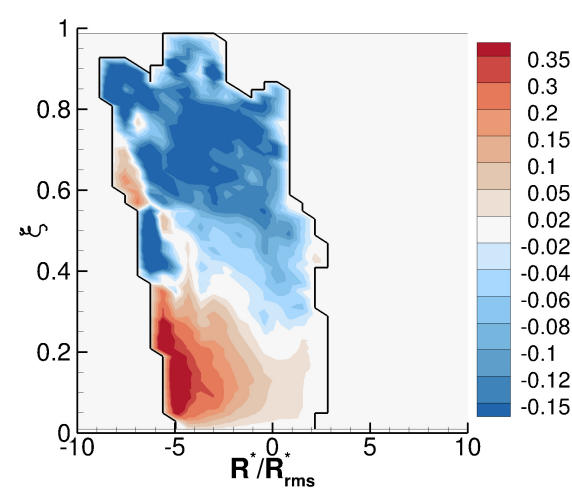

(b) $t_{M E}$, DNS

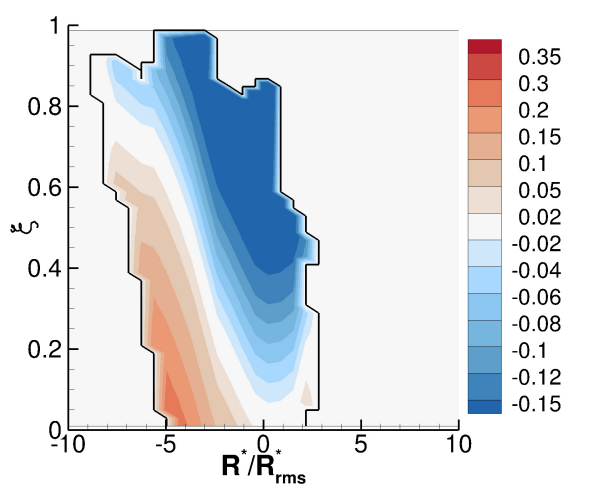

(e) $t_{M E}, \mathrm{SPMM}$

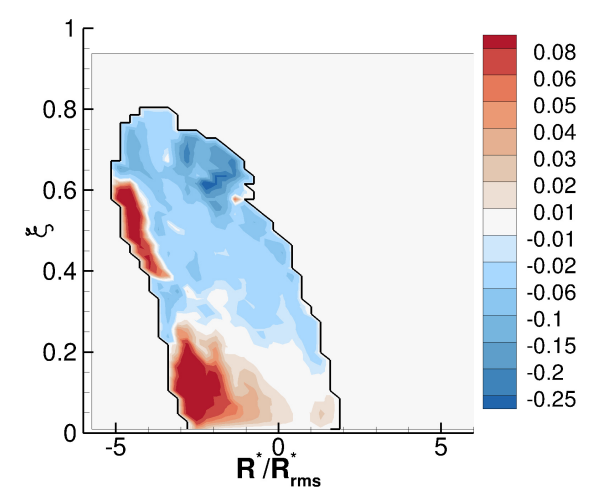

(c) $t_{R I}, \mathrm{DNS}$

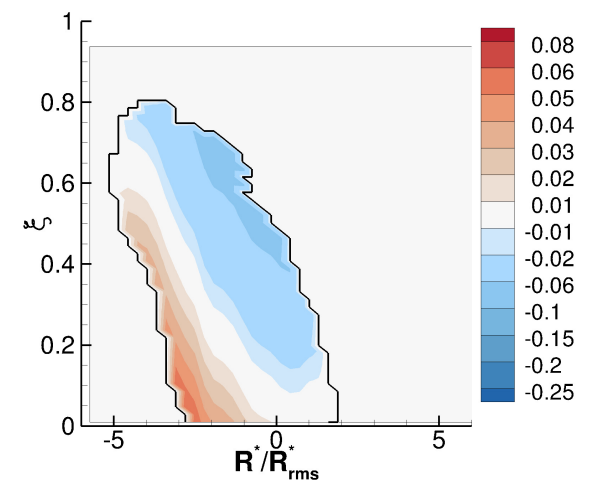

(f) $t_{R I}$, SPMM

Figure 16: Iso-contours of $D^{S P M M}(\xi) t_{j}$ (top rows) and versus $D_{M}^{S P M M}(\xi) t_{j}$ (bottom rows) at different time instants at $y_{P L}$. $R^{\star}$ is normalized by its corresponding r.m.s. at that time and location. 


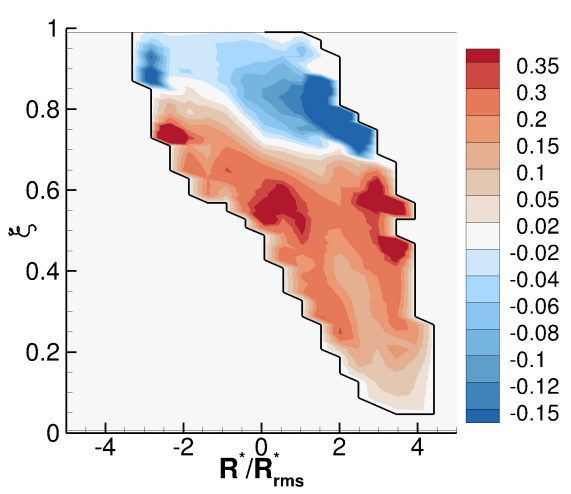

(a) $t_{B M E}$, DNS

留

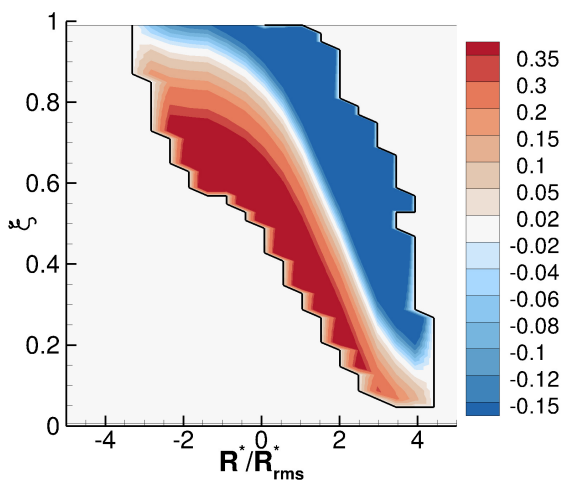

(d) $t_{B M E}$, SPMM

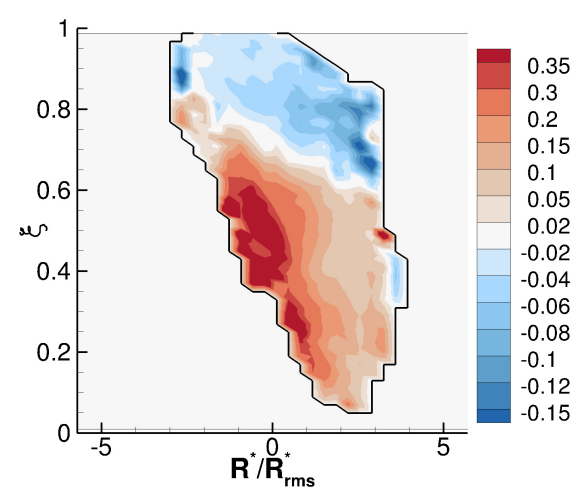

(b) $t_{M E}$, DNS

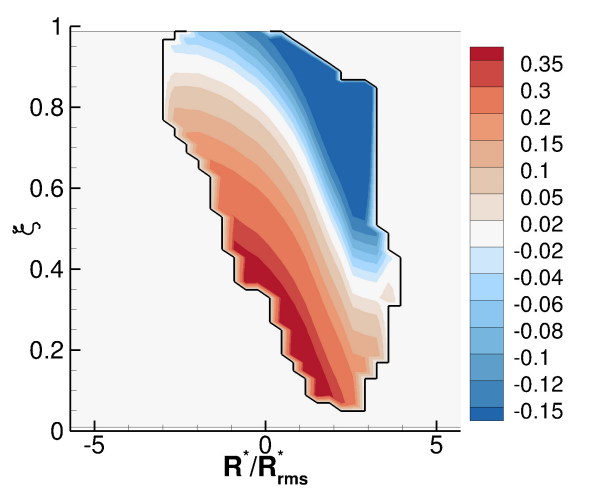

(e) $t_{M E}$, SPMM

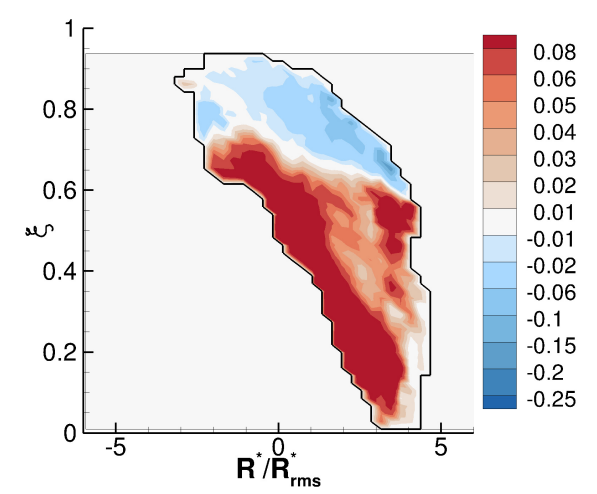

(c) $t_{R I}, \mathrm{DNS}$

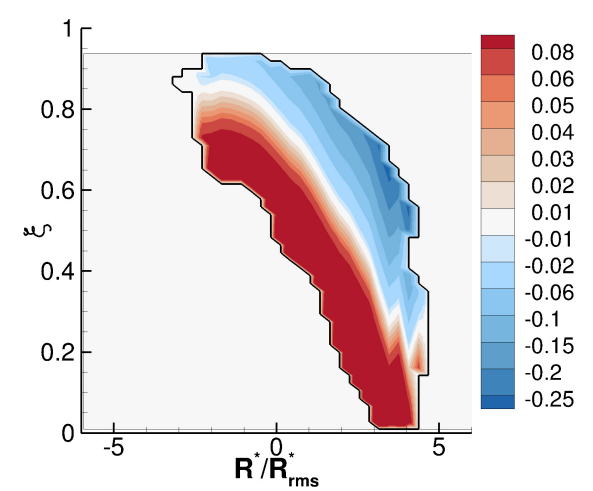

(f) $t_{R I}, \mathrm{SPMM}$

Figure 17: Iso-contours of $D^{S P M M}(\xi) t_{j}$ (top rows) and versus $D_{M}^{S P M M}(\xi) t_{j}$ (bottom rows) at different time instants at $y_{P R}$. $R^{\star}$ is normalized by its corresponding r.m.s. at that time and location. 


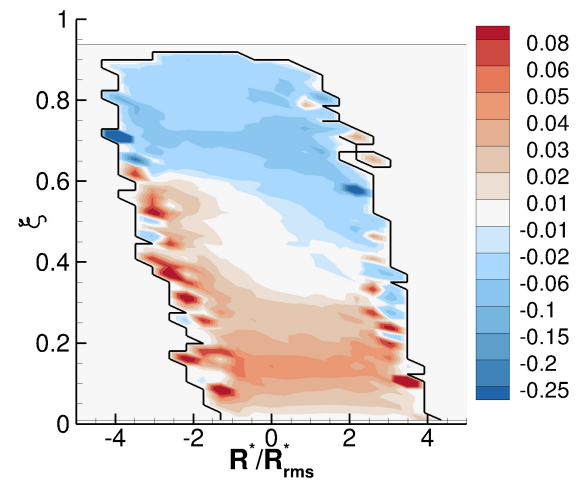

(a) $D^{S P M M}$, at $y_{P M}$

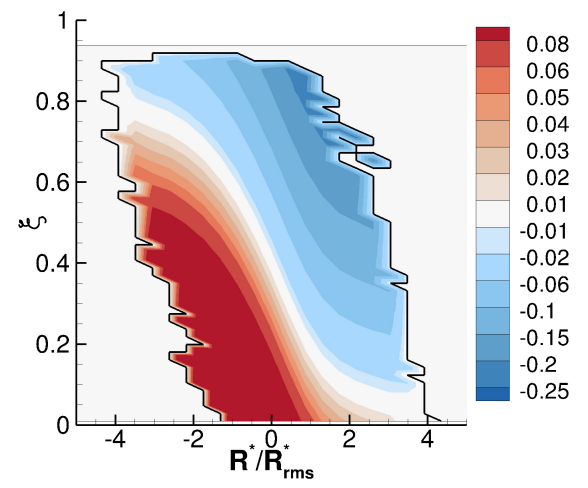

(c) $D_{M}^{S P M M}$, at $y_{P M}$

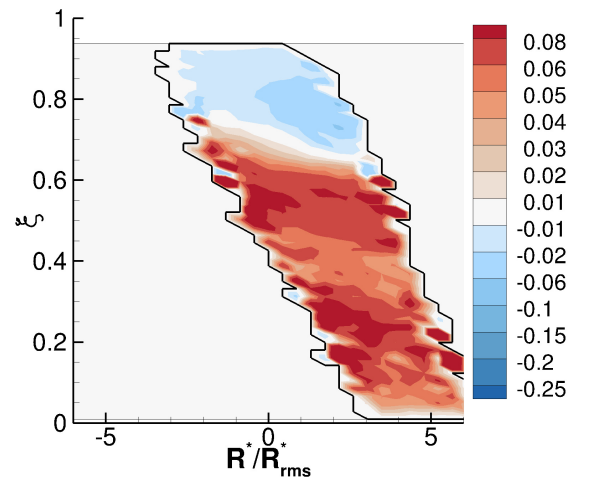

(b) $D^{S P M M}$, at $y_{P R}$

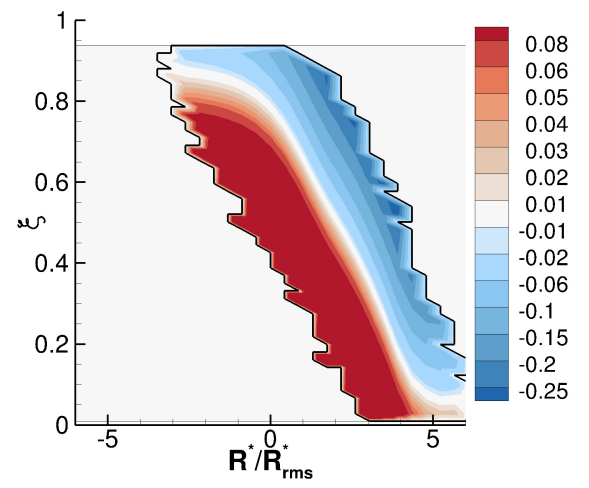

(d) $D_{M}^{S P M M}$, at $y_{P R}$

Figure 18: $D^{S P M M}(\xi) t_{j}$ (top rows) and $D_{M}^{S P M M}(\xi) t_{j}$ (bottom rows), based on $R_{s g}^{*}$ at $14 t_{j}$ at $y_{P M}$ and $y_{P R}$. 


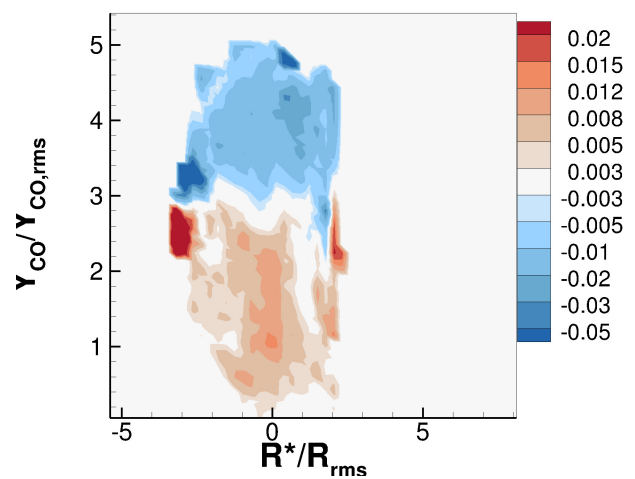

(a) $\mathrm{CO}, D^{S P M M}$

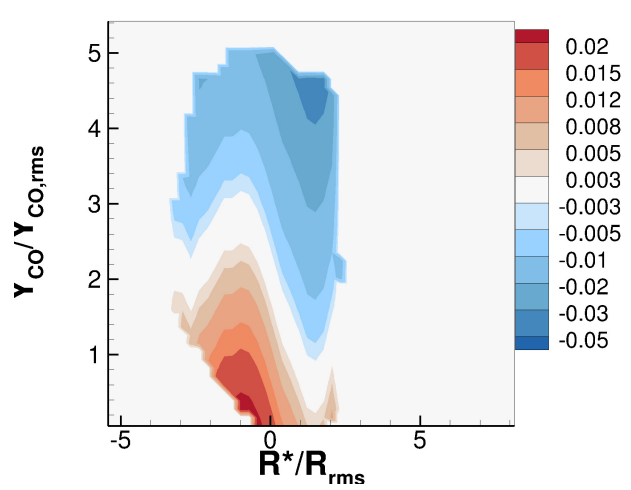

(c) $\mathrm{CO}, D_{M}^{S P M M}$

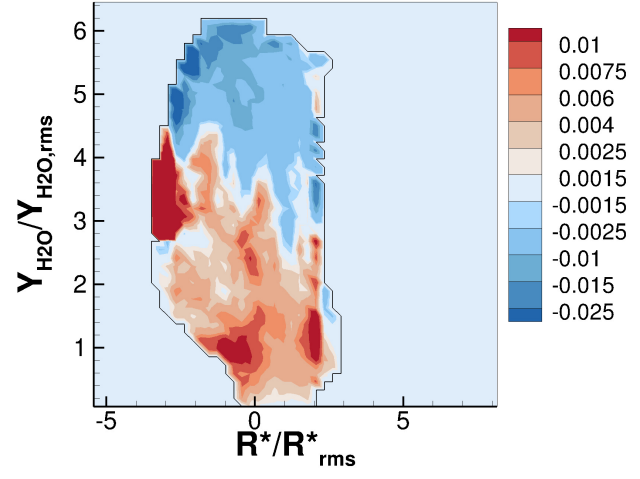

(b) $\mathrm{H}_{2} \mathrm{O}, D^{S P M M}$

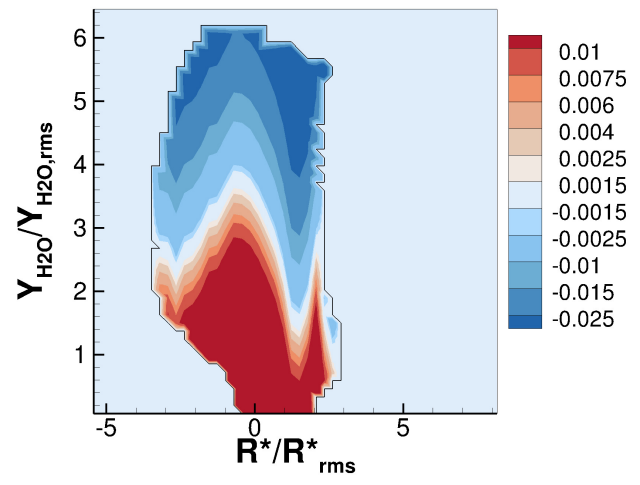

(d) $\mathrm{H}_{2} \mathrm{O}, D_{M}^{S P M M}$

Figure 19: $D^{S P M M^{2}} t_{j}$ (top rows) and $D_{M}^{S P M M} t_{j}$ (bottom rows) of $\mathrm{CO}$ and $\mathrm{H}_{2} \mathrm{O}$, based on Method 1 at $t_{R I}$ at $y_{P M}$. 


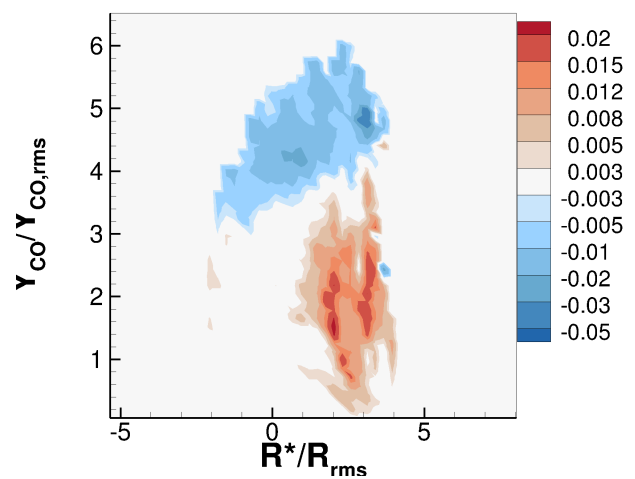

(a) $\mathrm{CO}, D^{S P M M}$

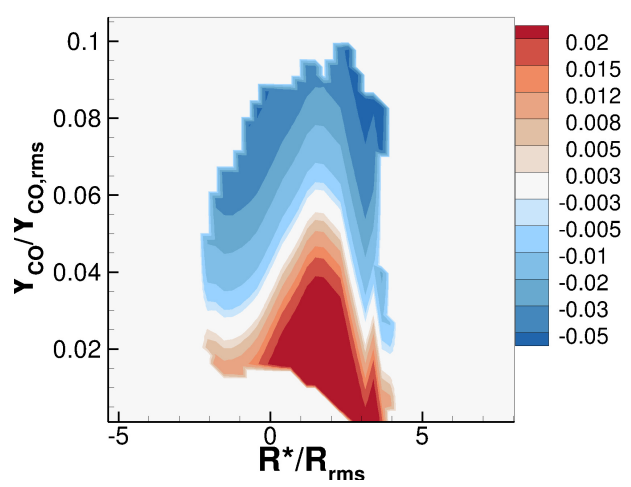

(c) $\mathrm{CO}, D_{M}^{S P M M}$

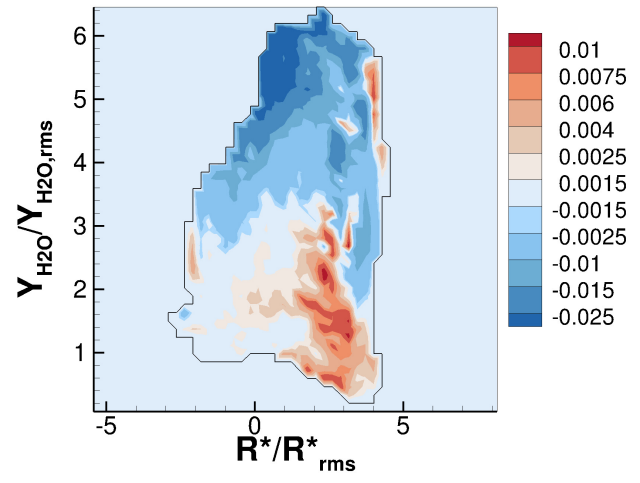

(b) $\mathrm{H}_{2} \mathrm{O}, D^{S P M M}$

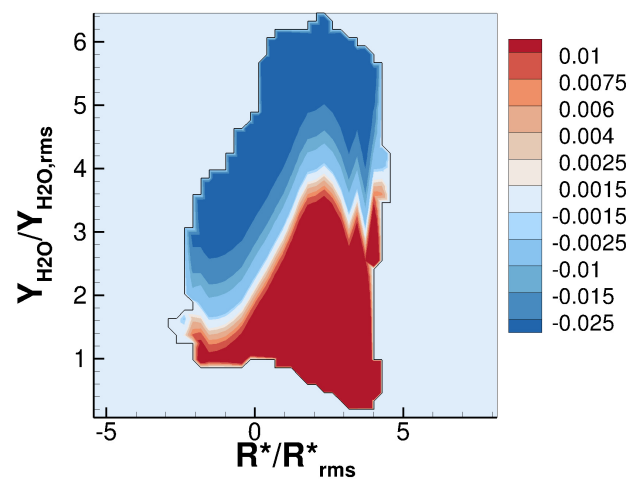

(d) $\mathrm{H}_{2} \mathrm{O}, D_{M}^{S P M M}$

Figure 20: $D^{S P M M^{2}} t_{j}$ (top rows) and $D_{M}^{S P M M}$ (bottom rows) of $\mathrm{CO}$ and $\mathrm{H}_{2} \mathrm{O}$, based on Method 1 at $t_{R I}$ at $y_{P R}$. 


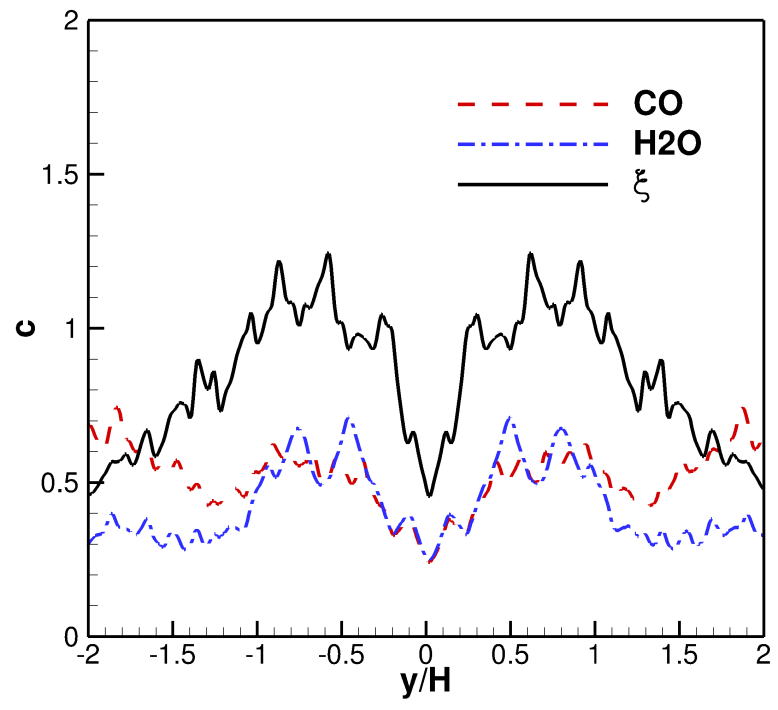

Figure 21: Comparison between values of $c$ obtained by matching scalar dissipation rates of $\mathrm{H}_{2} \mathrm{O}$, CO, and mixture fraction $\xi$ at $t_{R I}$ using Eq. 47, respectively. 


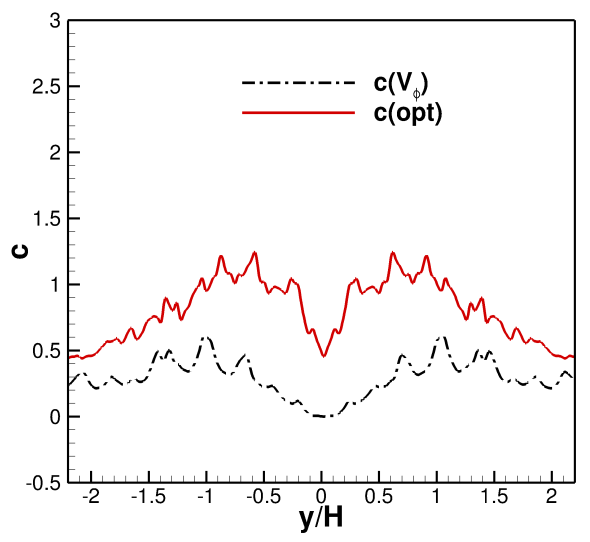

(c) $t_{R I}$

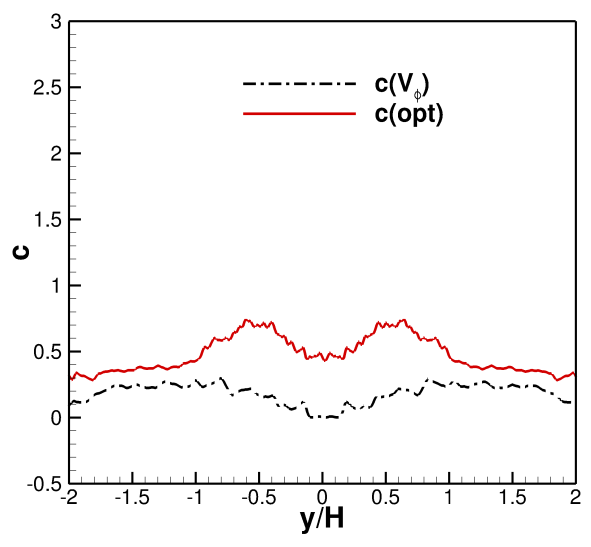

(b) $t_{M E}$

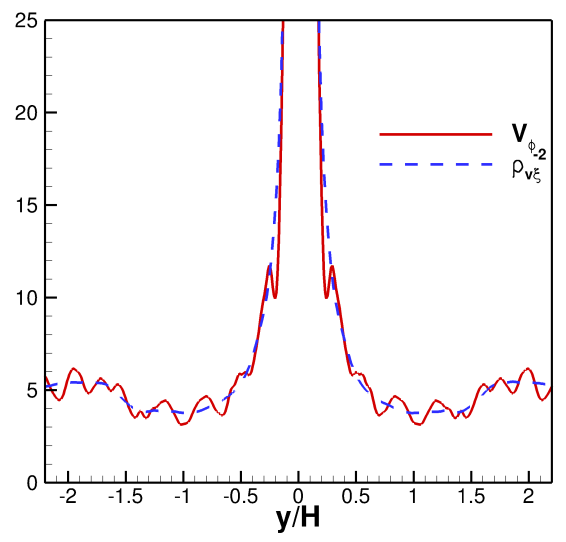

(d) $t_{R I}$

Figure 22: Profiles of $c$ calculated from Eq. $44\left(c\left(V_{\phi}\right)\right)$ versus $c$ calculated from Eq. 47 (c(opt)) at different time instants. $V_{\phi}$ and $\rho_{V \xi}^{-2}$ at $t_{R I}$ are shown in (d). 


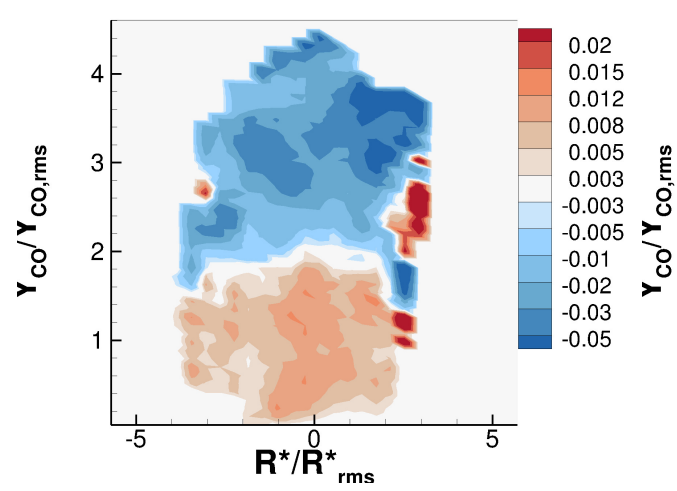

(a) $a=1$

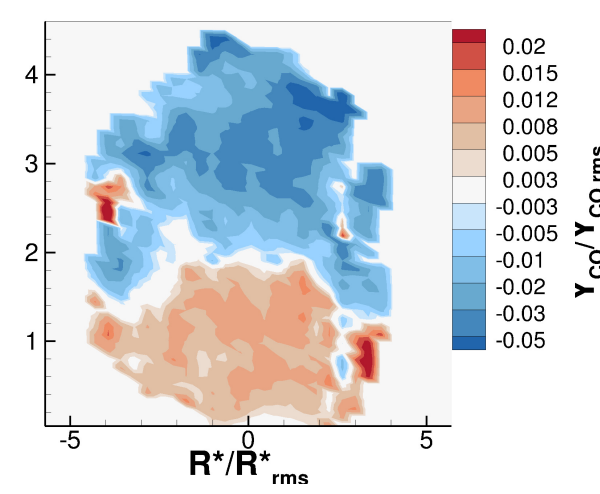

(b) $a=10$

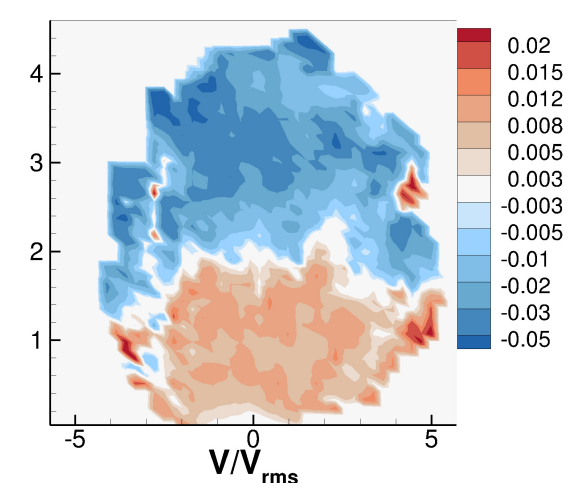

(c) IECM 